Provided for non-commercial research and education use. Not for reproduction, distribution or commercial use.

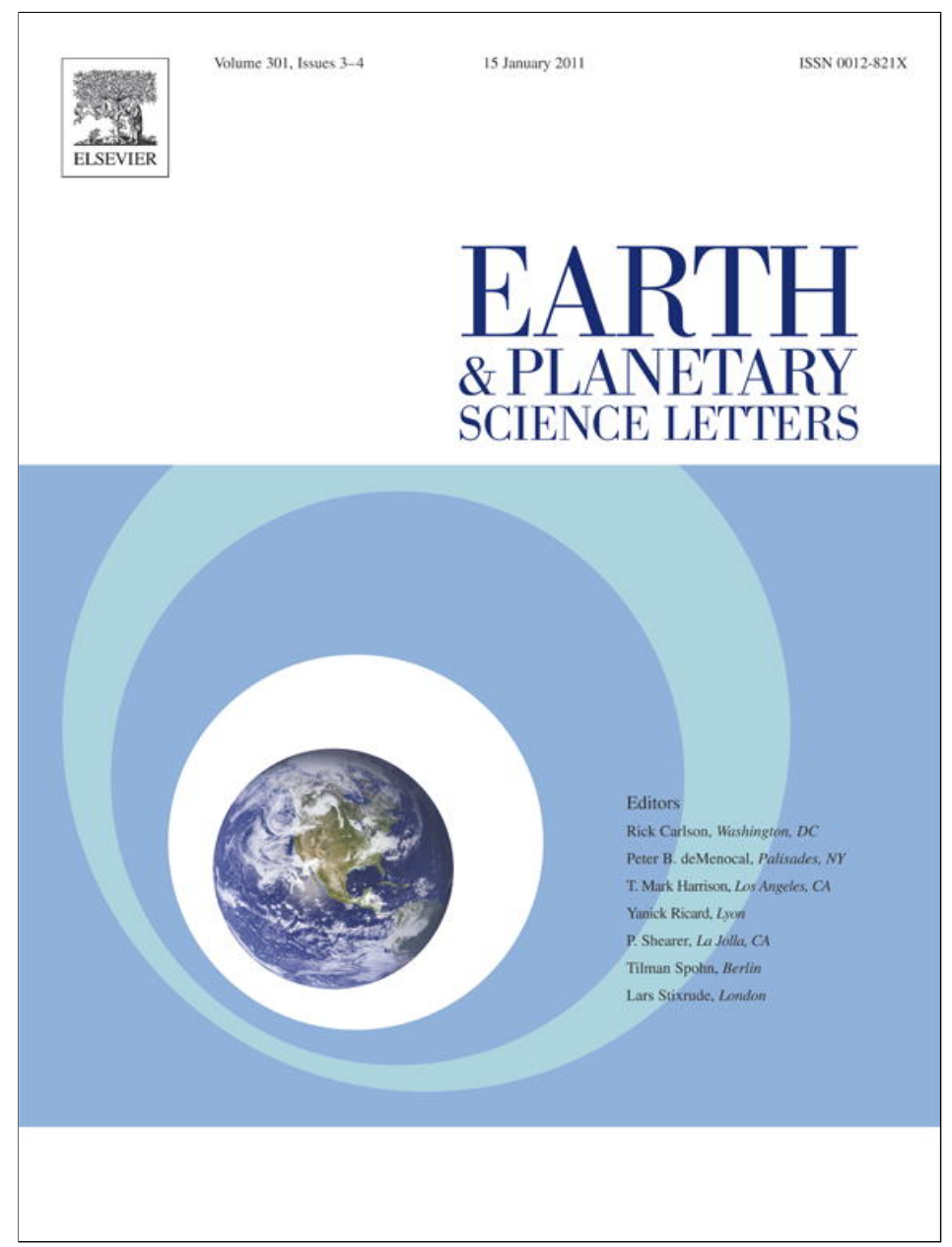

This article appeared in a journal published by Elsevier. The attached copy is furnished to the author for internal non-commercial research and education use, including for instruction at the authors institution and sharing with colleagues.

Other uses, including reproduction and distribution, or selling or licensing copies, or posting to personal, institutional or third party websites are prohibited.

In most cases authors are permitted to post their version of the article (e.g. in Word or Tex form) to their personal website or institutional repository. Authors requiring further information regarding Elsevier's archiving and manuscript policies are encouraged to visit:

http://www.elsevier.com/copyright 


\title{
The Th/La and Sm/La conundrum of the Tethyan realm lamproites
}

\author{
Simone Tommasini ${ }^{\mathrm{a}, *}$, Riccardo Avanzinelli ${ }^{\mathrm{a}}$, Sandro Conticelli ${ }^{\mathrm{a}, \mathrm{b}}$ \\ a Dipartimento di Scienze della Terra, Università degli Studi di Firenze, Via Giorgio La Pira 4, Firenze, I-50121, Italy \\ b Istituto di Geoscienze e Georisorse, Unità Operativa di Firenze, CNR, Via Giorgio La Pira 4, Firenze, I-50121, Italy
}

\section{A R T I C L E I N F O}

\section{Article history:}

Received 21 May 2010

Received in revised form 15 October 2010

Accepted 15 November 2010

Available online 17 December 2010

Editor: T.M. Harrison

\section{Keywords:}

Tethys oceans

lamproite

mantle metasomatism

trace elements

radiogenic isotopes

geodynamics

\begin{abstract}
A B S T R A C T
The Oligocene-Pleistocene Tethyan Realm Lamproites, from the Mediterranean to Himalayas, have a clear subduction-related signature and represent one of the most exotic and rare ultrapotassic mantle-derived magmas. They share the major element and mineralogical characteristics of the lamproite clan rocks, but clearly define a distinct subgroup with respect to within-plate lamproites on the basis of a number of key trace element ratios and radiogenic isotopes. The most striking characteristic of the Tethyan Realm Lamproites is the positive correlation between Th/La (up to >1.5) and Sm/La (up to >0.3), which is opposite to what observed in subduction-related magmas worldwide and cannot be reconciled with typical slab recycling processes. The geochemical conundrum of the Tethyan Realm Lamproites requires a component with high $\mathrm{Sm} / \mathrm{La}$ and Th/La (hence named SALATHO), in addition to a normal $\mathrm{K}_{2} \mathrm{O}$-rich sediment melt component (with low Th/La and $\mathrm{Sm} / \mathrm{La}$ ). The Pb isotope composition of the Tethyan Realm Lamproites also displays a mixing array from a normal sediment melt component to a high ${ }^{208} \mathrm{~Pb} /{ }^{206} \mathrm{~Pb}$ and low ${ }^{206} \mathrm{~Pb} /{ }^{204} \mathrm{~Pb}$ end-member that can be reconciled with the SALATHO component. This end-member requires a history of high timeintegrated $\kappa\left({ }^{232} \mathrm{Th} /{ }^{238} \mathrm{U}\right)$ and low time-integrated $\mu\left({ }^{238} \mathrm{U} /{ }^{204} \mathrm{~Pb}\right)$ and ought to be older than some hundreds of million years.

We propose a multi-stage process for the formation of the mantle sources of the Tethyan Realm Lamproites related to the tectonic mélange domains (i.e. chaotic mixture of depleted peridotite, basalt, and sediment) accreted to the Eurasia plate during the collisional events of the northward drifting continental slivers from Gondwana. In a first stage, the mélange domains experienced high $\mathrm{P}$ and low $\mathrm{T}$ metamorphism with segregation and stabilisation of lawsonite and zoisite/epidote veins, which potentially match the geochemical characteristics required by the SALATHO component. Successively, the subduction of the Neotethys and Alpine Tethys oceanic plates produced normal $\mathrm{K}_{2} \mathrm{O}$-rich sediment melts that migrated through the mantle wedge and metasomatised the depleted lithospheric mantle blocks within the chaotic mélange, forming a clinopyroxene-phlogopite vein network. Eventually, the Tertiary orogenic belt collapses triggered the onset of low-degree melting of the low-solidus fractions within the mélange domains, producing lamproitic magmas.
\end{abstract}

(c) 2010 Elsevier B.V. All rights reserved.

\section{Introduction}

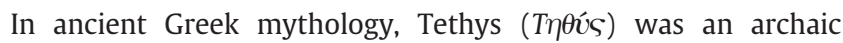
Titaness Sea Goddess, wife of the Sea God Oceanus, and mother of all the Oceanids. In the geologic literature too, Tethys is a sort of Mother of All Oceans prior to the Tertiary, and since its introduction in the 19th century (Suess, 1893), is a well-established term to define a series of ancient fossil ocean basins (Prototethys, Paleotethys, Neotethys, Alpine Tethys) separating the northern from the southern continents from Palaeozoic to Cenozoic (e.g. Gaetani et al., 2003;

\footnotetext{
* Corresponding author.

E-mail address: toms@unifi.it (S. Tommasini).
}

Scotese, 2004; Stampfli, 2000; Stampfli and Borel, 2002; and references therein). The Tethyan oceanic lithospheric domains, originally present between Eurasia and Gondwana, have been subducted and partially obducted, and remnants of these former Tethyan ocean basins are found as ophiolites outcropping along multiple narrow suture zones from the Western Mediterranean to Himalayan orogenic belts. The general structure of the $>18,000 \mathrm{~km}$ E-W striking Tethyan realm orogenic belts (Fig. 1), from Morocco and Spain to India and Bhutan has been described in a wealth of studies since the pioneering work of Argand (1924). This array of interconnected orogenic belts formed as a consequence of diachronous collisions of Gondwana-derived crustal plates rifted and northward drifted from the margin of Gondwana as three elongate continental slivers in the Silurian, Early Permian and Late Triassic-Late Jurassic. 


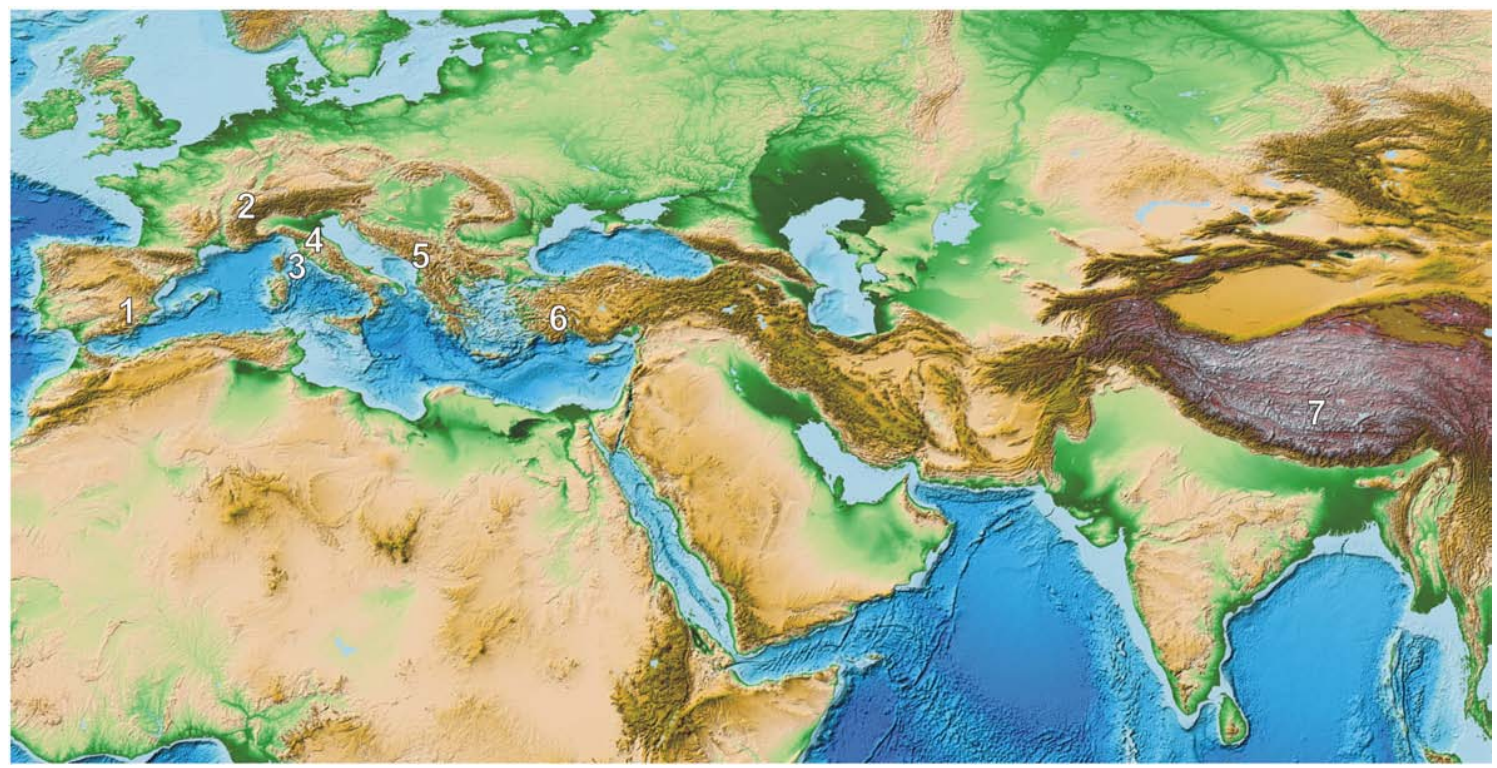

Fig. 1. Shaded relief image of the Tethyan realm orogenic belts from Spain to Southern Tibet, showing the main outcropping areas of the Tethyan realm lamproites from west to east: (1) Murcia-Almeria, Spain; (2) Western Alps, Italy; (3) Corsica, France; (4) Tuscany, Italy; (5) Vardar Zone, Serbia and Macedonia; (6) Western Anatolia, Turkey; (7) West Lhasa Terrane, China.

The prolonged subduction of the different Tethyan realm oceans along with the diachronous collision of the northward drifting continental slivers was accompanied by long-lived magmatic activity that is still active. The magmas produced represent one of the most impressive features of the geodynamic evolution of the Mediterranean-Himalayan orogenic belts, and are characterised by significant petrological, geochemical and isotopic variety ranging from subductionrelated signatures with calc-alkaline, shoshonite and ultrapotassic magmas, to within-plate signatures with OIB-like magmas (e.g. Altherr et al., 2004, 2008; Chung et al., 2005; Conticelli et al., 2009; Dilek and Altunkaynak, 2007; Duggen et al., 2004; Gao et al., 2007; Harangi et al., 2006; Innocenti et al., 2005; Lustrino and Wilson, 2007; Miller et al., 1999; Topuz et al., 2005; Turner et al., 1996; Williams et al., 2004; Wilson and Bianchini, 1999).

The Tethyan Realm Lamproites (TRL) represent minor and peculiar mantle-derived magmas of this widespread magmatic activity. They are ultrapotassic rocks extremely enriched in incompatible trace elements and formed by low-degree melting of depleted mantle sources metasomatised in subduction-related settings (e.g. Conticelli et al., 2007, 2009; Foley, 1992b; Peccerillo and Martinotti, 2006; Prelević and Foley, 2007; Prelević et al., 2005, 2008; and references therein).

This study is based upon a comprehensive database of the TRL most of the Western Mediterranean lamproites are authors' data (Conticelli et al., 2009) - and other worldwide lamproites, and we focus the discussion on some key trace element ratios $(\mathrm{Sm} / \mathrm{La}, \mathrm{Th} / \mathrm{La})$ and $\mathrm{Pb}$ isotopes that so far have been overlooked and represent something of a paradox: the TRL are characterised by high and positively correlated $\mathrm{Th} / \mathrm{La}$ and $\mathrm{Sm} / \mathrm{La}$ along with high ${ }^{208} \mathrm{~Pb} /{ }^{206} \mathrm{~Pb}$ for a given ${ }^{206} \mathrm{~Pb} /{ }^{204} \mathrm{~Pb}$. These unique geochemical and isotopic characteristics clearly separate the TRL from both within-plate lamproites and, most notably, subduction-related magmas worldwide.

The geochemical and isotopic paradox demands for a common genetic process affecting the mantle sources of the TRL, related to the formation of the Tethyan realm orogenic belts as a consequence of diachronous collisions of Gondwana-derived crustal plates since the Palaeozoic. This scenario opens new insights into the origin of the mantle source of subduction-related lamproites and bears also consequences to the geodynamic evolution of the Western Mediterranean orogenic belts.

\section{Geochemical background}

\subsection{The ultrapotassic rock clan}

On the basis of major element characteristics (Foley, 1992a), ultrapotassic volcanic rocks are classified as lamproites (Group I), kamafugites (Group II), and plagioleucitites (Group III). Lamproites have low $\mathrm{Al}_{2} \mathrm{O}_{3}, \mathrm{CaO}$ and $\mathrm{Na}_{2} \mathrm{O}$ contents, a silica range from 45 to 55 wt.\%, and high $\mathrm{Mg \#}[\mathrm{mol} \mathrm{Mg} /(\mathrm{Mg}+\mathrm{Fe})]$ and $\mathrm{K}_{2} \mathrm{O} / \mathrm{Al}_{2} \mathrm{O}_{3}$. Kamafugites have also low $\mathrm{Al}_{2} \mathrm{O}_{3}$ and $\mathrm{Na}_{2} \mathrm{O}$, but higher $\mathrm{CaO}$ and lower $\mathrm{SiO}_{2}$ than lamproites. Plagioleucitites have high $\mathrm{Al}_{2} \mathrm{O}_{3}$ and $\mathrm{Na}_{2} \mathrm{O}$, coupled with low $\mathrm{K}_{2} \mathrm{O} / \mathrm{Al}_{2} \mathrm{O}_{3}$, and intermediate $\mathrm{Mg} \#$ and $\mathrm{CaO}$ contents between lamproites and kamafugites. Experimental studies (e.g. Edgar, 1987; Edgar and Vukadinovic, 1992; Melzer and Foley, 2000; Wendlandt and Eggler, 1980a,b) demonstrated that different partial pressures of $\mathrm{H}_{2} \mathrm{O}$ and $\mathrm{CO}_{2}$ along with different mantle sources (amphibole/phlogopite harzburgite vs. wehrlite) play a significant role in producing lamproite vs. kamafugite magmas.

\subsection{The Tethyan realm lamproites vs. within-plate lamproites}

Lamproites represent one of the most exotic and rare mantlederived magmas. They occur in both within-plate and subductionrelated settings (e.g. Bergman, 1987). The TRL belong to the subductionrelated subgroup (e.g. Conticelli and Peccerillo, 1992; Conticelli et al., 2009; Gao et al., 2007; Miller et al., 1999; Prelević and Foley, 2007; Prelević et al., 2005, 2008; and references therein) and occur in a limited number of small-volume scattered outcrops of hypabyssal and volcanic rocks along the Tethyan realm orogenic belts from the Mediterranean to Southern Tibet (Fig. 1). The gap from Turkey to Southern Tibet could simply represent sampling bias due to areas poorly investigated (e.g. Iran, Afghanistan).

The TRL are invariably associated with calc-alkaline and shoshonitic rocks. Their ages range from Oligocene (Western Alps, NW Vardar Zone), to Miocene (West Lhasa Terrane, Corsica, Murcia-Almeria, Western Anatolia), Mio-Pliocene (Southern Vardar Zone), and PlioPleistocene (Tuscany) (Table 1). Their petrological and geochemical characteristics, along with their association with other subductionrelated magmas have been thoroughly discussed in a wealth of studies appropriately referenced by Conticelli et al. (2009), Gao et al. (2007), 
Peccerillo and Martinotti (2006), and Prelević and Foley (2007). The TRL are $\mathrm{SiO}_{2}$-rich (45-55 wt.\%), high $\mathrm{MgO}$ (9-12 wt.\%) ultrapotassic rocks that share a number of mineralogical and major element characteristics with within-plate lamproites worldwide (e.g. Bergman, 1987; Foley, 1992a). They are plagioclase-free rocks with high modal amount of phlogopite and forsteritic olivine, minor amount of Al-poor clinopyroxene and sanidine, and a number of accessory phases such as $\mathrm{K}$-richterite, apatite, pseudobrookite, picroilmenite. The most mafic rocks have $\mathrm{K}_{2} \mathrm{O}>3$ wt.\%, $\mathrm{CaO}<$ wt.\%, $\mathrm{Al}_{2} \mathrm{O}_{3}<12$ wt.\%, and $\mathrm{K}_{2} \mathrm{O} / \mathrm{Na}_{2} \mathrm{O}>2$. Other common characteristics of all lamproites worldwide are (i) the high enrichment in incompatible trace elements (Table 1), and (ii) the $\mathrm{Sr}, \mathrm{Nd}, \mathrm{Pb}$ isotope composition, which is among the most extreme of any mantle-derived magma (Fig. 2, Table 1 ).

The low $\mathrm{CaO}, \mathrm{Na}_{2} \mathrm{O}$, and $\mathrm{Al}_{2} \mathrm{O}_{3}$ contents along with the high-Fo olivine phenocrysts and associated $\mathrm{Cr}$-rich spinel inclusions provide compelling evidence for derivation of lamproites from a depleted lithospheric mantle source, whilst the extreme incompatible trace element enrichment, along with the $\mathrm{Sr}$ and $\mathrm{Nd}$ isotope composition, requires re-fertilisation of the mantle source by $\mathrm{K}_{2} \mathrm{O}$-rich metasomatic agents which permeated and reacted with the peridotitic mantle producing clinopyroxene/amphibole/phlogopite-rich veins (e.g. Edgar, 1987; Foley, 1992b; Melzer and Foley, 2000; Sekine and Wyllie, 1982).

The TRL have, however, a number of peculiar geochemical characteristics which make them unique in the lamproitic clan. First, they are clearly distinguished from within-plate lamproites worldwide (Western Australia, Leucite Hills, Smoky Butte, Gaussberg, Aldan Shield) on the basis of Sr-Nd (Fig. 2) and Pb isotope compositions (Table 1). Second, they have a clear subduction-related signature, exemplified by highly radiogenic $\mathrm{Sr}$ isotopes (Fig. 2), crust-like trace element patterns and High Field Strength Element depletion (Fig. 3), which provide compelling evidence for a crustal component recycled into the mantle via subduction zones, as the $\mathrm{K}_{2} \mathrm{O}$-rich metasomatic agent responsible for mantle re-fertilisation (e.g. Avanzinelli et al., 2009; Foley, 1992b; Peccerillo and Martinotti, 2006; Prelević and Foley, 2007; Prelević et al., 2008). The wide range of ${ }^{87} \mathrm{Sr} /{ }^{86} \mathrm{Sr}$ and ${ }^{143} \mathrm{Nd} /{ }^{144} \mathrm{Nd}$ (Fig. 2) indicates the involvement of crustal components of variable composition in the TRL mantle sources (Prelević et al., 2005). Despite the crust-like patterns, highly incompatible trace elements (Rb, Ba, Th, and U, Fig. 3) have on average different ratios with respect to present-day Upper Crust and Global Subducting Sediment (GLOSS, Plank and Langmuir, 1998), and represent a distinctive signature of the TRL. Third, on $\mathrm{Ba} / \mathrm{Rb}$ vs. $\mathrm{Rb} / \mathrm{Sr}$ and K/Th vs. Th (Fig. 4), the TRL form a separated group with respect to other lamproites. Their higher Rb/Sr coupled with lower Ba/Rb (Fig. 4a) provides arguments for the major role of phlogopite in their mantle sources with respect to the major role of amphibole in the case of other lamproites. Also, the TRL have generally lower K/Th than other lamproites (Fig. 4b): this is caused by the extreme Th enrichment, up to $>200$ ppm (Table 1 ), rather than lower $\mathrm{K}_{2} \mathrm{O}$ contents. Fourth, perhaps the most striking characteristic, they exhibit a positive correlation between Th/La and Sm/La (Fig. 5). The high Th/La (up to > 1.5) and Sm/La (up to $>0.3$ ) is caused by the combination of two factors: the extreme Th enrichment and the slightly concave downward LREE patterns (e.g. Conticelli et al., 2009) of the TRL. The extreme Th contents also result in abnormally high $\mathrm{Th} / \mathrm{U}(\mathrm{Th} / \mathrm{U}>10$, Table 1$)$, well beyond the $\mathrm{Th} / \mathrm{U} \leq 4$ of most crust and mantle rocks (e.g. McDonough, 1990; Plank and Langmuir, 1998; Rudnick and Gao, 2003; Sun and McDonough, 1989). The Th/U $>4$ is not unique to the TRL, being exhibited also by the Western Australia and Gaussberg lamproites (Table 1); in the TRL, however, the high $\mathrm{Th} / \mathrm{U}$ is coupled with high $\mathrm{Th} / \mathrm{Ta}$ (Table 1) reinforcing the subduction-related origin of the TRL and hence their different geodynamic setting with respect to within-plate lamproites.

\subsection{The Tethyan realm lamproites vs. subduction-related magmas}

The positive correlation between Th/La and Sm/La does not simply distinguish the TRL from within-plate lamproites (Fig. 5), it actually represents something of a paradox when compared to subductionrelated magmas worldwide. Magmas produced by depleted mantle sources (i.e. high Sm/La and low Th/La) that have been enriched in trace elements via sediment recycling in subduction zones exhibit a negative correlation on Th/La vs. Sm/La (grey field, Fig. 5), owing to the low Sm/La and relatively high Th/La ( 0.3-0.5) of the sediment component metasomatising the mantle wedge (Plank, 2005). In addition, modern volcanic arc magmas, as well as crustal rocks (Plank, 2005; Plank and Langmuir, 1998; Rudnick and Gao, 2003) have Th/ $\mathrm{La} \leq 0.5$, whilst the TRL have extreme values of Th/La (Fig. 5), which are not observed in any other mantle-derived magma. The negative correlation between Th/La and Sm/La in modern volcanic arcs is widely recognised as a key characteristic of arc magmas (Plank, 2005). Depleted mantle sources metasomatised by low T fluid-dominated components, form magmas lying at low Th/La and high $\mathrm{Sm} / \mathrm{La}$, whilst those metasomatised by high $\mathrm{T}$ melt-dominated components, form magmas with relatively high $\mathrm{Th} / \mathrm{La}$ - but well below unity - and low $\mathrm{Sm} / \mathrm{La}$ (grey field, Fig. 5), resembling the subducted sediment composition. In each volcanic arc setting, the similarity between Th/ La of subducted sediments and erupted magmas suggests no significant fractionation during slab and mantle melting (Plank, 2005).

The $\mathrm{K}_{2} \mathrm{O}$ enrichment and other subduction-related characteristics of the TRL have been widely interpreted as related to recycling of sedimentary material into their depleted lithospheric mantle sources (Conticelli and Peccerillo, 1992; Conticelli et al., 2007; Foley, 1992b; Peccerillo and Martinotti, 2006; Prelević and Foley, 2007; Prelević et al., 2005, 2008). However, it is important to point out that the high $\mathrm{Sm} / \mathrm{La}$ (and Th/La) of the TRL is not correlated to their subduction signature and $\mathrm{K}_{2} \mathrm{O}$ enrichment (Fig. 6), indicating the presence of a further metasomatic agent in the TRL mantle sources.

\section{The SALATHO component}

As a whole the TRL exhibit a positive mixing array from a normal crustal component to another component with high Sm/La and Th/La (hereafter SALATHO). Whilst the normal crustal component can be referred to typical $\mathrm{K}_{2} \mathrm{O}$-rich sediment melts of subduction zones, decoding the SALATHO component is problematic and we shall discuss it in detail. To a first approximation the SALATHO component should also have a broad crustal origin. The West Lhasa, Spain, Western Alps, and Tuscan lamproites, in which the SALATHO component is most evident (Table 1, Fig. 5), have extremely radiogenic $\mathrm{Sr}$ isotopes (Fig. 2) and trace element patterns similar to other TRL that are dominated by the normal sediment melt component (e.g. Western Anatolia and Corsica lamproites, Fig. 3). We therefore discuss the SALATHO component as deriving from a protolith dominated by sedimentary lithologies. It is worth pointing out that although both components (SALATHO and $\mathrm{K}_{2} \mathrm{O}$-rich sediment melts) are interpreted as sediment-derived, we shall see that they actually refer to two distinct and time-separated processes. In the following discussion we shall use present day GLOSS as a general proxy for sediments, due to the lack of detailed information on the composition of sediments deposited within each Tethyan realm ocean basin. Moreover, the use of specific sediment composition for each area would focus the discussion on local processes, in contrast with the common geochemical characteristics exhibited by the TRL (e.g. Fig. 5), suggesting a genetic link between these magmas.

The LREE and Th budget of slab-derived metasomatic agents delivered to the mantle wedge is controlled by accessory phases such as apatite, monazite, allanite (e.g. Hermann and Rubatto, 2009; Klimm et al., 2008; Plank, 2005; Skora and Blundy, 2010). Apatite has $\mathrm{D}_{\mathrm{Sm}}>\mathrm{D}_{\mathrm{La}}>\mathrm{D}_{\mathrm{Th}}$ (e.g. Prowatke and Klemme, 2006), meaning that any apatite in the sediment residue after dehydration/melting will determine the production of metasomatic agents with Th/La slightly higher and $\mathrm{Sm} / \mathrm{La}$ lower than the starting material. This can 
Table 1

Average composition of selected major and trace elements, radiogenic isotopes, and trace element ratios of the Tethyan realm lamproites and within-plate lamproites worldwide.

\begin{tabular}{|c|c|c|c|c|c|c|c|c|c|c|c|c|c|c|c|}
\hline $\begin{array}{l}\text { Nation } \\
\text { Locality } \\
\text { Orogenic Belt }\end{array}$ & Age & & $\mathrm{SiO}_{2}$ & $\mathrm{Al}_{2} \mathrm{O}_{3}$ & $\mathrm{MgO}$ & $\mathrm{CaO}$ & $\mathrm{K}_{2} \mathrm{O}$ & $\mathrm{P}_{2} \mathrm{O}_{5}$ & $\mathrm{Cr}$ & $\mathrm{Ni}$ & $\mathrm{Rb}$ & $\mathrm{Sr}$ & $\mathrm{Ba}$ & $\mathrm{La}$ & $\mathrm{Sm}$ \\
\hline \multicolumn{16}{|c|}{ Tethyan realm lamproites } \\
\hline Spain & Miocene & Avg & 54.8 & 10.5 & 10.6 & 4.1 & 6.9 & 1.0 & 649 & 451 & 470 & 677 & 1873 & 89 & 27.2 \\
\hline Murcia-Almeria & & $1 \mathrm{sd}$ & 3.4 & 1.6 & 3.5 & 2.1 & 1.8 & 0.3 & 165 & 145 & 223 & 287 & 653 & 19 & 5.5 \\
\hline \multirow[t]{3}{*}{ Betics } & & Min & 44.8 & 7.3 & 3.5 & 2.4 & 3.4 & 0.6 & 334 & 150 & 30 & 402 & 1182 & 59 & 17.4 \\
\hline & & Max & 60.5 & 14.5 & 19.8 & 11.9 & 9.5 & 2.1 & 941 & 714 & 903 & 1617 & 4334 & 143 & 40.2 \\
\hline & & $\mathrm{n}$ & 33 & 33 & 33 & 33 & 33 & 33 & 33 & 33 & 41 & 41 & 33 & 33 & 41 \\
\hline Italy & Oligocene & Avg & 52.9 & 11.0 & 9.9 & 5.8 & 7.6 & 1.1 & 564 & 280 & 433 & 797 & 3728 & 102 & 29.8 \\
\hline Western Alps & & $1 \mathrm{sd}$ & 3.5 & 1.1 & 2.0 & 1.6 & 1.7 & 0.1 & 189 & 81 & 128 & 156 & 616 & 30 & 6.8 \\
\hline \multirow[t]{3}{*}{ Alps } & & Min & 48.8 & 8.9 & 7.0 & 3.1 & 3.7 & 0.8 & 309 & 93 & 158 & 530 & 2308 & 54 & 15.6 \\
\hline & & Max & 61.7 & 13.5 & 13.6 & 8.4 & 9.8 & 1.4 & 933 & 460 & 646 & 1242 & 4740 & 164 & 39.4 \\
\hline & & $\mathrm{n}$ & 17 & 17 & 17 & 17 & 17 & 17 & 17 & 17 & 17 & 17 & 15 & 13 & 12 \\
\hline France & Miocene & Avg & 58.1 & 10.7 & 6.7 & 3.3 & 10.5 & 0.7 & 422 & 267 & 340 & 764 & 1194 & 172 & 17.7 \\
\hline Corsica & & $1 \mathrm{sd}$ & 0.7 & 0.1 & 0.3 & 0.3 & 0.2 & 0.1 & 56 & 31 & 25 & 92 & 199 & 26 & 1.6 \\
\hline \multirow[t]{3}{*}{ Alps } & & Min & 56.7 & 10.5 & 6.4 & 2.9 & 10.2 & 0.6 & 340 & 230 & 317 & 640 & 925 & 135 & 16.3 \\
\hline & & Max & 58.6 & 10.8 & 7.1 & 3.7 & 10.7 & 0.8 & 530 & 322 & 376 & 847 & 1450 & 193 & 19.1 \\
\hline & & $\mathrm{n}$ & 7 & 7 & 7 & 7 & 7 & 7 & 7 & 7 & 7 & 7 & 7 & 7 & 4 \\
\hline Italy & Pliocene & Avg & 57.2 & 12.3 & 8.2 & 4.0 & 7.6 & 0.8 & 502 & 247 & 580 & 528 & 1174 & 104 & 23.7 \\
\hline Tuscany & & $1 \mathrm{sd}$ & 1.3 & 1.2 & 1.4 & 1.0 & 0.4 & 0.3 & 85 & 84 & 146 & 132 & 143 & 36 & 3.8 \\
\hline \multirow[t]{3}{*}{ Apennines } & & Min & 55.3 & 10.6 & 4.2 & 2.2 & 7.0 & 0.5 & 380 & 92 & 371 & 236 & 760 & 46 & 18.4 \\
\hline & & Max & 62.0 & 14.6 & 11.8 & 6.9 & 8.9 & 1.3 & 672 & 427 & 874 & 686 & 1400 & 173 & 29.0 \\
\hline & & $\mathrm{n}$ & 30 & 30 & 30 & 30 & 30 & 30 & 18 & 30 & 30 & 30 & 30 & 30 & 9 \\
\hline Serbia \& Macedonia & Oligocene & Avg & 49.8 & 11.8 & 8.9 & 7.3 & 5.2 & 1.1 & 488 & 284 & 239 & 975 & 2140 & 67 & 13.7 \\
\hline Vardar Zone & Mio-Pliocene & $1 \mathrm{sd}$ & 3.1 & 1.5 & 2.0 & 1.6 & 1.4 & 0.4 & 263 & 140 & 90 & 342 & 994 & 27 & 4.8 \\
\hline \multirow{3}{*}{ Dinarides } & & $\min$ & 42.4 & 9.7 & 4.3 & 3.8 & 2.8 & 0.5 & 65 & 45 & 72 & 394 & 410 & 23 & 6.5 \\
\hline & & Max & 60.1 & 15.3 & 13.4 & 9.9 & 7.4 & 1.9 & 1176 & 653 & 554 & 1712 & 4189 & 135 & 21.9 \\
\hline & & $\mathrm{n}$ & 56 & 56 & 56 & 56 & 56 & 56 & 56 & 56 & 56 & 56 & 56 & 54 & 49 \\
\hline Turkey & Miocene & Avg & 49.1 & 10.6 & 10.1 & 8.1 & 6.6 & 1.4 & 610 & 353 & 279 & 1516 & 2017 & 109 & 12.4 \\
\hline Western Anatolia & & $1 \mathrm{sd}$ & 2.3 & 1.3 & 2.2 & 1.8 & 0.8 & 0.3 & 130 & 180 & 123 & 597 & 659 & 50 & 2.1 \\
\hline \multirow[t]{3}{*}{ Taurides } & & Min & 45.7 & 8.9 & 5.6 & 4.9 & 4.8 & 0.6 & 159 & 49 & 189 & 476 & 249 & 43 & 8.6 \\
\hline & & Max & 54.2 & 13.3 & 12.9 & 10.7 & 8.5 & 2.1 & 792 & 597 & 726 & 2326 & 3242 & 188 & 16.4 \\
\hline & & $\mathrm{n}$ & 25 & 25 & 25 & 25 & 25 & 25 & 21 & 25 & 25 & 25 & 25 & 15 & 14 \\
\hline China & Miocene & Avg & 55.4 & 12.8 & 6.1 & 5.7 & 7.3 & 1.0 & 353 & 191 & 655 & 942 & 2851 & 130 & 35.9 \\
\hline West Lhasa Terrane & & $1 \mathrm{sd}$ & 2.0 & 0.8 & 1.2 & 1.1 & 1.0 & 0.2 & 80 & 54 & 146 & 262 & 537 & 30 & 7.8 \\
\hline \multirow[t]{3}{*}{ Tibetan Plateau } & & Min & 53.3 & 10.9 & 3.5 & 3.9 & 4.5 & 0.6 & 239 & 87 & 391 & 621 & 1863 & 72 & 24.6 \\
\hline & & Max & 60.7 & 14.3 & 10.2 & 7.1 & 8.6 & 1.4 & 565 & 358 & 939 & 1633 & 3931 & 200 & 48.0 \\
\hline & & $\mathrm{n}$ & 29 & 29 & 29 & 29 & 29 & 29 & 29 & 29 & 29 & 29 & 29 & 29 & 29 \\
\hline \multicolumn{16}{|c|}{ Worldwide within-plate lamproites } \\
\hline \multirow[t]{5}{*}{ Gaussberg } & Pleistocene & Avg & 50.6 & 9.9 & 8.0 & 4.4 & 11.7 & 1.5 & 307 & 240 & 289 & 1610 & 5078 & 181 & 14.9 \\
\hline & & $1 \mathrm{sd}$ & 1.0 & 0.3 & 0.4 & 0.2 & 0.4 & 0.1 & 38 & 38 & 40 & 125 & 298 & 12 & 0.9 \\
\hline & & Min & 48.7 & 9.4 & 7.1 & 4.1 & 11.0 & 1.4 & 218 & 154 & 160 & 1355 & 4302 & 154 & 11.9 \\
\hline & & Max & 52.4 & 10.4 & 8.8 & 4.7 & 12.5 & 1.6 & 365 & 339 & 323 & 1816 & 5686 & 204 & 16.4 \\
\hline & & $\mathrm{n}$ & 24 & 24 & 24 & 24 & 24 & 24 & 24 & 24 & 24 & 24 & 24 & 24 & 24 \\
\hline Leucite Hills & Pleistocene & Avg & 48.1 & 8.7 & 9.0 & 8.3 & 8.3 & 2.2 & 443 & 196 & 249 & 3855 & 7530 & 292 & 25.1 \\
\hline & & $1 \mathrm{sd}$ & 4.7 & 0.9 & 1.8 & 3.4 & 2.2 & 0.5 & 91 & 54 & 58 & 1555 & 2077 & 114 & 9.3 \\
\hline & & Min & 41.4 & 7.2 & 5.7 & 3.3 & 4.7 & 1.3 & 266 & 104 & 130 & 1830 & 4471 & 119 & 12.8 \\
\hline & & Max & 55.8 & 10.1 & 12.2 & 12.7 & 11.9 & 3.0 & 560 & 333 & 460 & 7233 & 12500 & 421 & 39.1 \\
\hline & & $\mathrm{n}$ & 40 & 40 & 40 & 40 & 40 & 40 & 40 & 40 & 40 & 40 & 40 & 40 & 33 \\
\hline Australia & Oligocene & Avg & 51.3 & 7.4 & 11.7 & 6.0 & 8.3 & 1.1 & 700 & 550 & 343 & 1350 & 9280 & 264 & 18.7 \\
\hline & Miocene & $1 \mathrm{sd}$ & 6.6 & 2.4 & 7.5 & 0.8 & 2.9 & 0.6 & & & 129 & 465 & 3933 & 86 & 6.4 \\
\hline & & Min & & & & & & & & & 190 & 549 & 4400 & 110 & 7.6 \\
\hline & & Max & & & & & & & & & 614 & 2349 & 18100 & 425 & 29.3 \\
\hline & & $\mathrm{n}$ & & & & & & & & & 15 & 15 & 14 & 15 & 15 \\
\hline Smoky butte & Oligocene & Avg & 52.3 & 8.9 & 8.2 & 4.6 & 7.9 & 2.1 & 495 & 344 & 96 & 2706 & 11275 & 374 & 37.3 \\
\hline & & $1 \mathrm{sd}$ & 0.8 & 0.2 & 0.3 & 0.2 & 0.8 & 0.3 & 65 & & 34 & 364 & 4349 & 41 & 4.1 \\
\hline & & Min & 51.4 & 8.6 & 7.8 & 4.4 & 6.5 & 1.6 & 437 & & 50 & 2226 & 8709 & 332 & 32.6 \\
\hline & & Max & 53.5 & 9.1 & 8.5 & 4.9 & 8.8 & 2.3 & 583 & & 140 & 3150 & 20000 & 437 & 43.1 \\
\hline & & $\mathrm{n}$ & 6 & 6 & 6 & 6 & 6 & 6 & 6 & & 6 & 6 & 6 & 6 & 6 \\
\hline Aldan & Early & Avg & 45.9 & 6.9 & 16.4 & 7.4 & 5.7 & 0.8 & 826 & 393 & 192 & 1802 & 3823 & 32 & 6.4 \\
\hline & Cretaceous & $1 \mathrm{sd}$ & 1.9 & 1.8 & 5.6 & 1.5 & 2.1 & 0.7 & 443 & 301 & 80 & 1386 & 3679 & 23 & 3.4 \\
\hline & & $\min$ & 42.4 & 4.6 & 7.3 & 4.2 & 2.9 & 0.3 & 110 & 73 & 87 & 561 & 1234 & 9 & 2.5 \\
\hline & & Max & 48.1 & 9.2 & 22.7 & 9.2 & 8.3 & 2.2 & 1515 & 900 & 314 & 4139 & 12773 & 68 & 12.9 \\
\hline & & $\mathrm{n}$ & 10 & 10 & 10 & 10 & 10 & 10 & 10 & 9 & 10 & 10 & 10 & 10 & 10 \\
\hline
\end{tabular}

Avg: average value, 1sd: absolute standard deviation, Min: minimum value, Max: maximum value, n: number of samples. Data source: Altherr et al. (2004); Benito et al. (1999); Bergman (1987); Coban and Flower (2007); Conticelli et al. (2009); Cvetković et al. (2004); Davies et al. (2006); Ersoy and Helvaci (2007); Francalanci et al. (2000); Fraser et al. (1985); Gao et al. (2007); Innocenti et al. (2005); Miller et al. (1999); Mirnejad and Bell (2006); Mitchell et al. (1987); Murphy et al. (2002); Nelson et al. (1986); Owen (2008); Prelević et al. (2004, 2005, 2008); Vollmer et al. (1984); and references therein. The complete lamproite clan database is available as supplementary material at doi:10.1016/j. epsl.2010.11.023.

potentially determine the typical negative correlation between $\mathrm{Th} / \mathrm{La}$ and $\mathrm{Sm} / \mathrm{La}$ as commonly observed in volcanic arc magmas (grey field, Fig. 5), but cannot account for the positive correlation exhibited by the TRL (Fig. 5).
The potential role of allanite and monazite has been assessed using a number of recent experimental studies on oceanic crust (basalt + sediment) dehydration and melting at P-T conditions relevant to subarc regions, in which either allanite or monazite was present (or 


\begin{tabular}{|c|c|c|c|c|c|c|c|c|c|c|c|c|c|c|}
\hline \multirow[t]{2}{*}{ Ta } & \multirow[t]{2}{*}{ Th } & \multirow[t]{2}{*}{$\mathrm{U}$} & \multirow{2}{*}{$\frac{{ }^{87} \mathrm{Sr}}{{ }^{86} \mathrm{Sri}}$} & \multirow{2}{*}{$\frac{{ }^{143} \mathrm{Nd}}{{ }^{144} \mathrm{Ndi}}$} & \multirow{2}{*}{$\frac{{ }^{206} \mathrm{~Pb}}{{ }^{204} \mathrm{Pbi}}$} & \multirow{2}{*}{$\frac{{ }^{207} \mathrm{~Pb}}{{ }^{204} \mathrm{Pbi}}$} & \multirow{2}{*}{$\frac{{ }^{208} \mathrm{~Pb}}{{ }^{204} \mathrm{Pbi}}$} & \multirow[t]{2}{*}{$\mathrm{Th} / \mathrm{La}$} & \multirow[t]{2}{*}{$\mathrm{Ba} / \mathrm{Rb}$} & \multirow[t]{2}{*}{$\mathrm{Rb} / \mathrm{Sr}$} & $\mathrm{K} / \mathrm{Th}$ & $\mathrm{Sm} / \mathrm{La}$ & $\mathrm{Th} / \mathrm{U}$ & $\mathrm{Th} / \mathrm{Ta}$ \\
\hline & & & & & & & & & & & & & & \\
\hline 2.86 & 101.2 & 21.3 & 0.7184 & 0.51203 & 18.78 & 15.72 & 39.11 & 1.1 & 5.2 & 0.79 & 582 & 0.32 & 5.1 & 37.0 \\
\hline 0.59 & 21.6 & 5.7 & 0.0025 & 0.00002 & 0.05 & 0.02 & 0.08 & 0.1 & 6.9 & 0.41 & 148 & 0.03 & 2.3 & 7.6 \\
\hline 2.03 & 64.0 & 8.5 & 0.7115 & 0.51197 & 18.66 & 15.68 & 38.98 & 0.8 & 2.0 & 0.05 & 255 & 0.26 & 3.4 & 23.8 \\
\hline 3.95 & 159.7 & 34.5 & 0.7226 & 0.51207 & 18.84 & 15.78 & 39.30 & 1.5 & 39.2 & 1.46 & 921 & 0.38 & 15.3 & 53.5 \\
\hline 31 & 33 & 37 & 33 & 33 & 28 & 28 & 28 & 33 & 33 & 41 & 33 & 33 & 33 & 31 \\
\hline 2.02 & 142.5 & 25.5 & 0.7168 & 0.51203 & 18.62 & 15.69 & 39.02 & 1.4 & 9.5 & 0.56 & 451 & 0.31 & 5.6 & 68.9 \\
\hline 0.39 & 35.2 & 5.2 & 0.0024 & 0.00004 & 0.06 & 0.02 & 0.22 & 0.2 & 3.3 & 0.22 & 98 & 0.03 & 1.0 & 16.9 \\
\hline 1.41 & 81.9 & 15.2 & 0.7121 & 0.51199 & 18.50 & 15.67 & 38.76 & 1.0 & 5.5 & 0.24 & 353 & 0.26 & 4.1 & 46.8 \\
\hline 2.73 & 226.0 & 33.6 & 0.7217 & 0.51210 & 18.72 & 15.72 & 39.50 & 1.8 & 14.6 & 1.17 & 728 & 0.35 & 8.1 & 108.7 \\
\hline 14 & 17 & 16 & 14 & 12 & 12 & 12 & 12 & 13 & 15 & 17 & 17 & 12 & 16 & 14 \\
\hline 3.54 & 36.0 & 12.8 & 0.71237 & 0.51215 & 18.82 & 15.70 & 39.31 & 0.2 & 3.5 & 0.45 & 5197 & 0.11 & 6.1 & 8.4 \\
\hline 0.57 & 16.6 & 16.0 & 0.0002 & & 0.05 & 0.01 & 0.02 & 0.1 & 0.6 & 0.03 & 7607 & 0.01 & 4.0 & 5.1 \\
\hline 3.00 & 4.3 & 4.3 & 0.71226 & 0.51215 & 18.79 & 15.69 & 39.29 & 0.03 & 2.9 & 0.40 & 1590 & 0.10 & 0.1 & 1.4 \\
\hline 4.10 & 53.0 & 36.8 & 0.71256 & 0.51215 & 18.85 & 15.70 & 39.32 & 0.3 & 4.6 & 0.50 & 20715 & 0.12 & 8.6 & 13.7 \\
\hline 4 & 6 & 4 & 3 & 2 & 2 & 2 & 2 & 6 & 7 & 7 & 6 & 4 & 4 & 4 \\
\hline 2.47 & 102.2 & 14.9 & 0.7161 & 0.51211 & 18.66 & 15.67 & 39.01 & 0.9 & 2.2 & 1.23 & 684 & 0.23 & 5.7 & 39.3 \\
\hline 0.37 & 26.5 & 3.0 & 0.0004 & 0.00002 & 0.03 & 0.03 & 0.11 & 0.3 & 0.6 & 0.57 & 247 & 0.05 & 1.4 & 13.9 \\
\hline 2.00 & 50.4 & 10.7 & 0.7158 & 0.51209 & 18.62 & 15.64 & 38.90 & 0.5 & 0.9 & 0.57 & 513 & 0.18 & 4.3 & 20.4 \\
\hline 3.10 & 121.0 & 18.2 & 0.7168 & 0.51212 & 18.69 & 15.69 & 39.15 & 1.5 & 3.5 & 2.46 & 1229 & 0.32 & 8.0 & 58.0 \\
\hline 9 & 14 & 7 & 11 & 4 & 4 & 4 & 4 & 14 & 30 & 30 & 14 & 9 & 7 & 9 \\
\hline 1.32 & 36.3 & 7.3 & 0.7095 & 0.51230 & 18.76 & 15.65 & 38.95 & 0.6 & 10.8 & 0.28 & 1370 & 0.20 & 6.2 & 28.4 \\
\hline 0.47 & 14.6 & 3.8 & 0.0014 & 0.00012 & 0.08 & 0.14 & 0.13 & 0.2 & 8.6 & 0.18 & 634 & 0.04 & 3.9 & 16.4 \\
\hline 0.77 & 15.0 & 1.9 & 0.7075 & 0.51213 & 18.58 & 15.12 & 38.78 & 0.0 & 1.6 & 0.07 & 611 & 0.13 & 2.5 & 11.0 \\
\hline 3.01 & 76.5 & 17.5 & 0.7116 & 0.51252 & 18.89 & 15.74 & 39.24 & 0.9 & 38.4 & 1.41 & 3132 & 0.26 & 18.0 & 72.2 \\
\hline 29 & 55 & 55 & 36 & 36 & 31 & 31 & 31 & 54 & 56 & 56 & 55 & 49 & 55 & 29 \\
\hline 5.41 & 17.2 & 6.4 & 0.7067 & 0.51237 & & & & 0.20 & 7.9 & 0.22 & 3376 & 0.13 & 3.0 & 4.3 \\
\hline 2.83 & 4.1 & 2.0 & 0.0025 & 0.00019 & & & & 0.10 & 3.1 & 0.14 & 862 & 0.05 & 0.9 & 3.1 \\
\hline 1.23 & 10.0 & 2.5 & 0.7038 & 0.51223 & & & & 0.05 & 0.8 & 0.09 & 1886 & 0.07 & 1.9 & 1.6 \\
\hline 10.00 & 28.0 & 10.0 & 0.7103 & 0.51250 & & & & 0.44 & 16.3 & 0.70 & 5446 & 0.22 & 5.3 & 15.4 \\
\hline 24 & 25 & 16 & 6 & 2 & & & & 15 & 25 & 25 & 25 & 14 & 16 & 24 \\
\hline 2.78 & 171.4 & 21.3 & 0.7235 & 0.51188 & 18.67 & 15.74 & 39.67 & 1.4 & 4.6 & 0.75 & 365 & 0.29 & 8.5 & 67.8 \\
\hline 0.94 & 34.2 & 5.0 & 0.0069 & 0.00006 & 0.17 & 0.05 & 0.15 & 0.4 & 1.5 & 0.26 & 80 & 0.06 & 2.3 & 24.4 \\
\hline 1.30 & 112.0 & 9.6 & 0.7166 & 0.51180 & 18.41 & 15.68 & 39.42 & 0.7 & 2.7 & 0.24 & 274 & 0.15 & 4.6 & 33.4 \\
\hline 4.54 & 231.7 & 29.4 & 0.7363 & 0.51200 & 18.93 & 15.84 & 40.06 & 2.2 & 8.8 & 1.14 & 549 & 0.38 & 13.7 & 130.0 \\
\hline 29 & 29 & 29 & 19 & 18 & 23 & 23 & 23 & 29 & 29 & 29 & 29 & 29 & 29 & 29 \\
\hline 4.99 & 23.2 & 3.2 & 0.7096 & 0.51192 & 17.50 & 15.60 & 38.26 & 0.13 & 18.0 & 0.18 & 4245 & 0.082 & 7.4 & 4.7 \\
\hline 0.25 & 2.8 & 0.4 & 0.0002 & 0.00004 & 0.05 & 0.02 & 0.08 & 0.01 & 3.4 & 0.03 & 573 & 0.004 & 1.0 & 0.6 \\
\hline 4.41 & 15.9 & 2.2 & 0.7092 & 0.51187 & 17.42 & 15.57 & 38.15 & 0.10 & 15.5 & 0.10 & 3373 & 0.077 & 4.6 & 2.9 \\
\hline 5.42 & 28.3 & 3.8 & 0.7099 & 0.51202 & 17.61 & 15.63 & 38.43 & 0.15 & 29.7 & 0.21 & 6056 & 0.093 & 9.5 & 5.8 \\
\hline 24 & 24 & 24 & 24 & 24 & 24 & 24 & 24 & 24 & 24 & 24 & 24 & 24 & 24 & 24 \\
\hline 5.09 & 36.2 & 8.5 & 0.70570 & 0.51195 & 17.38 & 15.48 & 37.35 & 0.12 & 32.8 & 0.08 & 2513 & 0.10 & 4.2 & 7.3 \\
\hline 1.96 & 12.9 & 2.8 & 0.0004 & 0.00011 & 0.14 & 0.01 & 0.14 & 0.01 & 14.2 & 0.04 & 2085 & 0.02 & 0.6 & 1.6 \\
\hline 2.00 & 13.7 & 3.4 & 0.70534 & 0.51177 & 17.18 & 15.46 & 37.13 & 0.11 & 10.2 & 0.02 & 904 & 0.06 & 3.1 & 4.9 \\
\hline 8.81 & 48.0 & 11.4 & 0.70779 & 0.51211 & 17.58 & 15.51 & 37.52 & 0.15 & 70.4 & 0.17 & 6968 & 0.17 & 5.2 & 10.8 \\
\hline 16 & 16 & 16 & 48 & 42 & 24 & 24 & 24 & 16 & 40 & 40 & 16 & 33 & 16 & 16 \\
\hline 8.08 & 32.9 & 4.1 & 0.7169 & 0.51188 & 17.44 & 15.73 & 38.16 & 0.11 & 29.9 & 0.28 & 2392 & 0.071 & 9.7 & 3.7 \\
\hline 3.10 & 19.1 & 2.9 & 0.0031 & 0.00013 & 0.16 & 0.03 & 0.32 & 0.04 & 10.8 & 0.14 & 1655 & 0.005 & 6.3 & 1.0 \\
\hline 4.68 & 0.0 & 0.0 & 0.7110 & 0.51166 & 17.23 & 15.68 & 37.20 & 0.07 & 12.7 & 0.16 & 687 & 0.064 & 2.6 & 2.5 \\
\hline 15.90 & 69.1 & 10.3 & 0.7206 & 0.51210 & 17.88 & 15.80 & 38.59 & 0.24 & 50.0 & 0.65 & 6286 & 0.081 & 31.5 & 6.0 \\
\hline 15 & 27 & 27 & 15 & 15 & 19 & 19 & 19 & 15 & 14 & 15 & 15 & 15 & 25 & 15 \\
\hline 5.81 & 6.5 & 1.9 & 0.7061 & 0.51138 & 16.24 & 15.22 & 36.39 & 0.017 & 126.7 & 0.04 & 10387 & 0.100 & 4.0 & 1.1 \\
\hline 0.64 & 1.0 & 1.1 & 0.0002 & 0.00008 & 0.25 & 0.04 & 0.20 & 0.001 & 50.1 & 0.02 & 2379 & 0.001 & 1.1 & 0.1 \\
\hline 5.08 & 5.3 & 1.1 & 0.7058 & 0.51127 & 16.03 & 15.19 & 36.20 & 0.016 & 73.9 & 0.02 & 8077 & 0.098 & 1.8 & 1.0 \\
\hline 6.79 & 7.8 & 4.0 & 0.7063 & 0.51150 & 16.64 & 15.28 & 36.68 & 0.019 & 191.0 & 0.05 & 13872 & 0.101 & 4.8 & 1.2 \\
\hline 6 & 6 & 6 & 6 & 6 & 6 & 6 & 6 & 6 & 6 & 6 & 6 & 6 & 6 & 6 \\
\hline 0.40 & 4.0 & 1.7 & 0.7065 & 0.51167 & 17.06 & 15.37 & 37.32 & 0.14 & 22.5 & 0.17 & 22321 & 0.24 & 2.6 & 12.3 \\
\hline 0.43 & 3.6 & 1.6 & 0.0008 & 0.00022 & 0.32 & 0.09 & 0.14 & 0.06 & 21.6 & 0.15 & 25192 & 0.09 & 0.7 & 6.1 \\
\hline 0.09 & 0.8 & 0.2 & 0.7055 & 0.51135 & 16.61 & 15.24 & 37.13 & 0.03 & 5.4 & 0.04 & 5481 & 0.15 & 1.9 & 4.8 \\
\hline 1.40 & 12.5 & 5.7 & 0.7079 & 0.51193 & 17.42 & 15.47 & 37.50 & 0.23 & 77.9 & 0.51 & 84592 & 0.45 & 3.9 & 23.8 \\
\hline 10 & 10 & 10 & 10 & 10 & 10 & 10 & 10 & 10 & 10 & 10 & 10 & 10 & 10 & 10 \\
\hline
\end{tabular}

has been inferred to be) as by-product (e.g. Hermann and Rubatto, 2009; Johnson and Plank, 1999; Kessel et al., 2005; Klimm et al., 2008; Skora and Blundy, 2010). In Figure 7, the Th/La and Sm/La of each experimental run have been normalised to those of the starting materials to cancel out trace element doping, whilst the trace element ratios of the TRL have been normalised to those of GLOSS in order to make a reasonable comparison between experimental runs and real magmas. Although the latter normalisation might not be strictly 


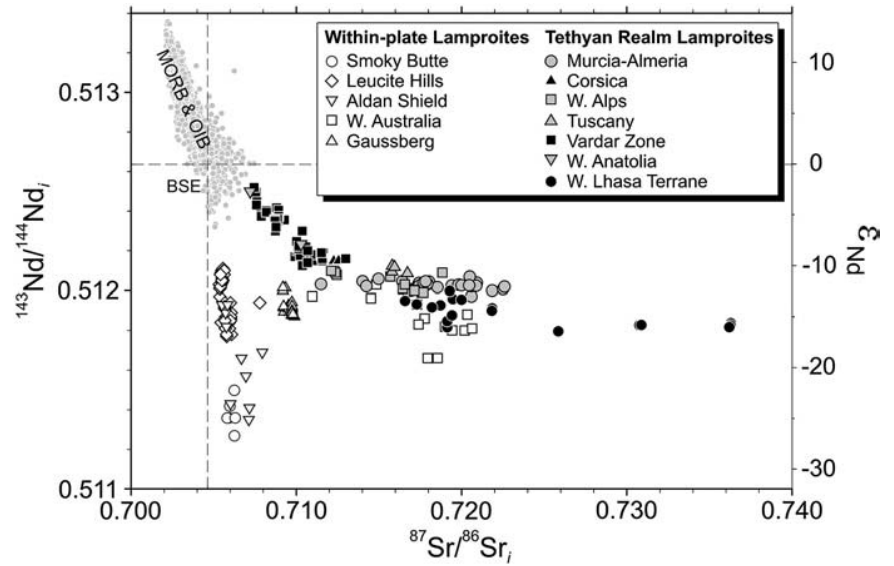

Fig. $2 .{ }^{87} \mathrm{Sr} /{ }^{86} \mathrm{Sr}_{i}$ vs. ${ }^{143} \mathrm{Nd} /{ }^{144} \mathrm{Nd}_{i}$ of rocks belonging to the lamproite clan. The TRL are clearly distinguished from MORBs, OIBs and within-plate lamproites, with the exception of the Western Australia lamproites. Lamproite data source as in Table 1, MORB-OIB data source from the compilation of Stracke et al. (2003); BSE: Bulk Silicate Earth.

correct, any other crustal composition would yield broadly similar results. Most of the metasomatic agents (either fluids, supercritical liquids, or melts) released during these experiments have $(\mathrm{Th} / \mathrm{La})_{\mathrm{n}}$ similar to that of the starting material in agreement with the negligible Th/La fractionation between arc magmas and subducted sediments (Plank, 2005). A number of experimental runs have (Th/ $\mathrm{La})_{\mathrm{n}}$ of about 2 , although invariably have $(\mathrm{Sm} / \mathrm{La})_{\mathrm{n}}<1$. In contrast, the TRL depict a positive trend towards $(\mathrm{Th} / \mathrm{La})_{\mathrm{n}}>8$ and $(\mathrm{Sm} / \mathrm{La})_{\mathrm{n}}>1.5$ which is not observed in any experimental run.

The TRL magmas with high Th/La and Sm/La have also high Th/U (see full data compilation in the supplementary material). In contrast, allanite/monazite saturated liquids always have enrichment of $U$ with respect to Th (Klimm et al., 2008; Skora and Blundy, 2010). Indeed Klimm et al. (2008) indicated allanite-saturated liquids as suitable candidates to produce the ${ }^{238} \mathrm{U}$-excesses observed in several volcanic arc magmas.

The corollary of these studies is that the ordinary metasomatic agents released during subduction, including those saturated in either allanite or monazite, are unable to produce mantle enrichment zones

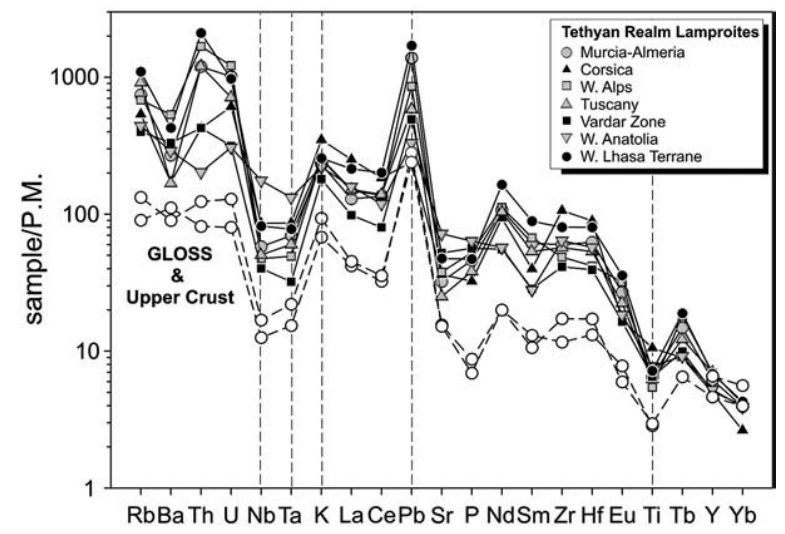

Fig. 3. Primitive mantle normalised (Sun and McDonough, 1989) incompatible element patterns of the average composition of the different outcropping localities of the TRL. The negative anomalies in $\mathrm{Nb}-\mathrm{Ta}$ and $\mathrm{Ti}$, along with the positive anomalies at $\mathrm{K}$ and $\mathrm{Pb}$ mimic the pattern of crustal material (Global Subducting Sediment, GLOSS, Plank and Langmuir, 1998; Upper Crust, Rudnick and Gao, 2003), and demonstrate the clear subduction-related signature of the TRL. Note the distinctive signature of the TRL in highly incompatible trace element ratios ( $\mathrm{Rb}, \mathrm{Ba}, \mathrm{Th}, \mathrm{U})$ in comparison to GLOSS and UC. Lamproite data source as in Table 1.
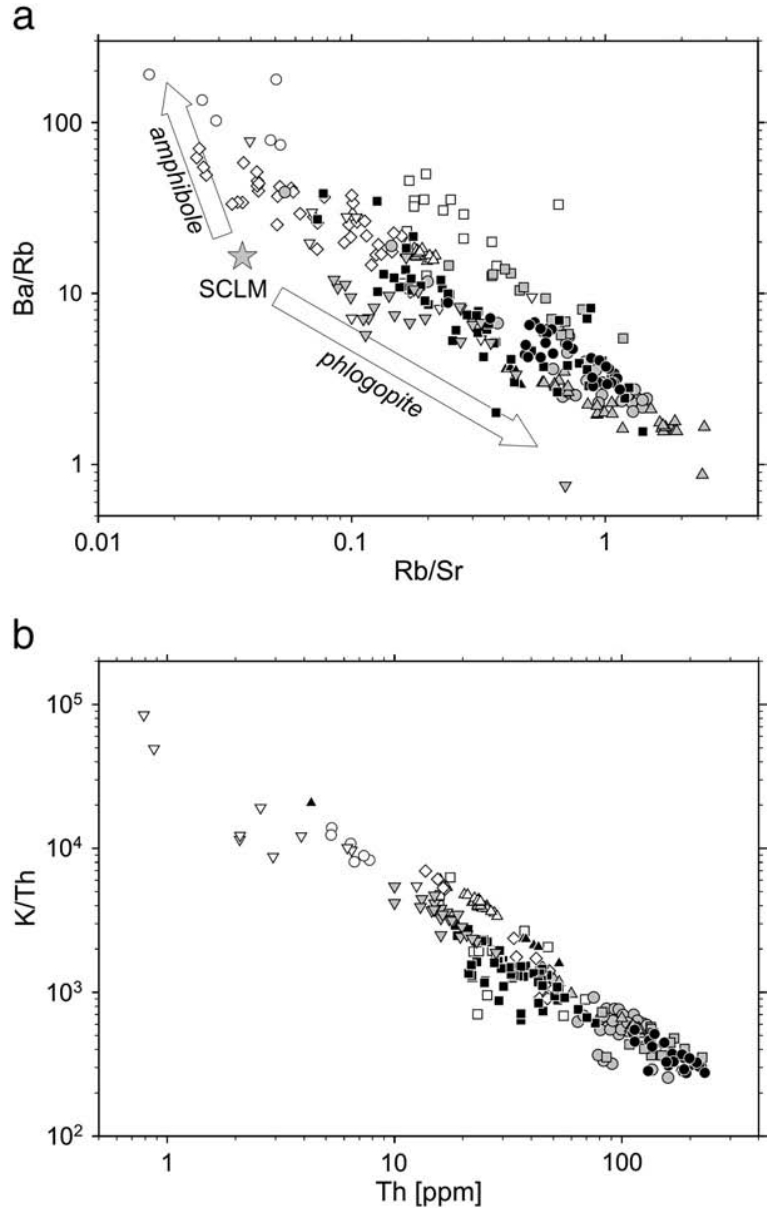

Fig. 4. $\mathrm{Ba} / \mathrm{Rb}$ vs. $\mathrm{Rb} / \mathrm{Sr}$ (a), and $\mathrm{K} / \mathrm{Th}$ vs. Th (b) of the lamproite clan rocks. (a) The TRL have on average higher $\mathrm{Rb} / \mathrm{Sr}$ and lower $\mathrm{Ba} / \mathrm{Sr}$ than within-plate lamproites suggesting a major role of phlogopite in their mantle source with respect to the major role of amphibole in the case of other lamproites. The Subcontinental Lithospheric Mantle composition (SCLM, McDonough, 1990) is reported for comparison along with qualitative arrows evidencing the expected compositional variation due to amphibole vs. phlogopite mantle metasomatism. (b) The TRL have lower K/Th than other lamproites due to their extreme Thenrichment up to $>200 \mathrm{ppm}$. Lamproite data source as in Table 1, symbols as in Figure 2.

and successively magmas with $\mathrm{Th} / \mathrm{La}>1, \mathrm{Sm} / \mathrm{La}>0.3$ and $\mathrm{Th} / \mathrm{U}>4$. This is actually not surprising considering that allanite and monazite are widely regarded as key minerals in controlling the Th, $U$ and REE budget of typical subduction-related magmas (e.g. Hermann and Rubatto, 2009; Klimm et al., 2008; Skora and Blundy, 2010), from which the TRL are clearly distinguished (Fig. 5).

We are thus left to face the apparent inconsistency of the Th/La and $\mathrm{Sm} / \mathrm{La}$ conundrum of the TRL, which requires a mantle source consisting of crust-like and depleted lithospheric mantle domains: the former being responsible for their extreme trace element contents, crust-like trace element patterns and radiogenic isotope $(\mathrm{Sr}, \mathrm{Nd}, \mathrm{Pb})$ signature, whilst the latter for their major element depleted flavour. However, the positive Th/La vs. Sm/La array delineated by the TRL (Fig. 5 and Fig. 7) suggests the presence of two distinct crustal components: (i) a normal $\mathrm{K}_{2} \mathrm{O}$-rich sediment melt with $(\mathrm{Sm} / \mathrm{La})_{\mathrm{n}}<1$ and $(\mathrm{Th} / \mathrm{La})_{\mathrm{n}} \sim 1$ (Fig. 7), similar to the metasomatic agents typically released during slab dehydration and melting and consistent with the composition of experimental runs; (ii) a SALATHO component with $(\mathrm{Sm} / \mathrm{La})_{\mathrm{n}}>1.5$ and $(\mathrm{Th} / \mathrm{La})_{\mathrm{n}}>8$, related to a process not generally occurring in (or recorded by) typical subduction-related geodynamic settings, but yet affecting the TRL mantle sources all along the $>18000 \mathrm{~km}$ E-W striking Tethyan realm orogenic belts. 


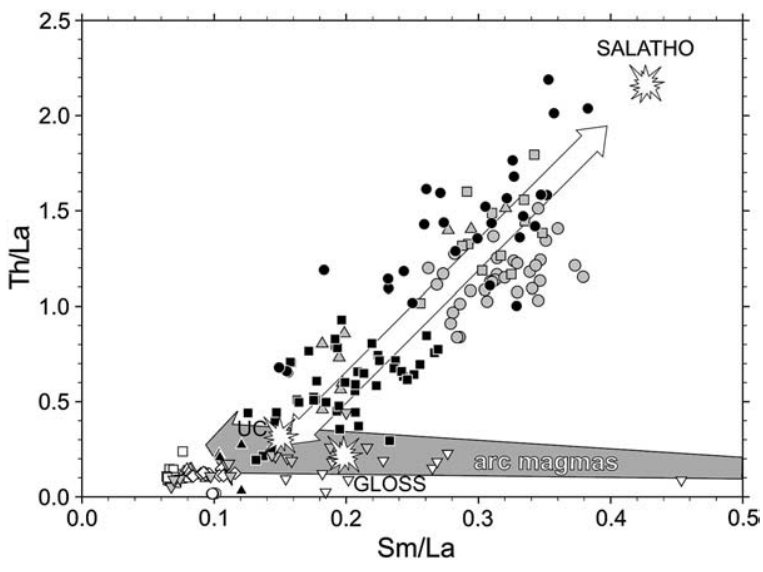

Fig. 5. Sm/La vs. Th/La of the TRL and within-plate lamproites, evidencing the positive correlation of the TRL along with their extreme trace element ratios. Upper Crust (UC, Rudnick and Gao, 2003) and Global Subducting Sediment (GLOSS, Plank and Langmuir, 1998) are reported as proxies of crustal recycled components. The grey field indicates the observed compositional variations of volcanic arc rocks related to slab-derived mantle metasomatism (Plank, 2005). The TRL plot on a mixing array (double-headed arrow) between a general crustal component similar to GLOSS (low Sm/La and Th/La), and a high Sm/La and Th/La component (hereafter SALATHO, see text). Lamproite data source as in Table 1, symbols as in Figure 2.

\subsection{The message from $\mathrm{Pb}$ isotopes}

The TRL have a broad positive correlation between Th/La and ${ }^{208} \mathrm{~Pb} /{ }^{206} \mathrm{~Pb}$, departing from the normal sediment melt end-member (approximated by GLOSS, Fig. 8) and forming a fan-shaped data scatter toward high Th/La. This suggests that the SALATHO component could have played a dominant role in controlling the time-integrated $\mathrm{Th} / \mathrm{U} / \mathrm{Pb}$, and hence the ${ }^{208} \mathrm{~Pb} /{ }^{206} \mathrm{~Pb}$ isotope composition of the TRL. This time-dependent signature could set constraints on the timing of formation of the unique characteristics of the TRL.

The TRL deviates systematically from the global array delineated by OIBs, MORBs, and other lamproites worldwide on ${ }^{206} \mathrm{~Pb} /{ }^{204} \mathrm{~Pb}$ vs. ${ }^{208} \mathrm{~Pb} /{ }^{206} \mathrm{~Pb}$ diagram (Fig. 9). They form a mixing array from a sediment melt end-member (approximated by GLOSS) to another end-member characterised by ${ }^{208} \mathrm{~Pb} /{ }^{206} \mathrm{~Pb}$ higher than the global array of mantle-derived magmas for a given ${ }^{206} \mathrm{~Pb} /{ }^{204} \mathrm{~Pb}$. This latter end-member, corresponding to the SALATHO component, requires a

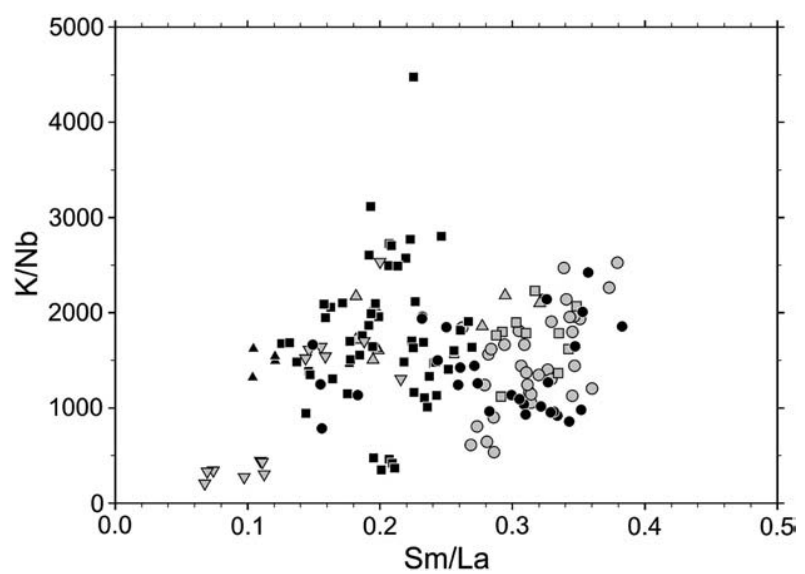

Fig. 6. $\mathrm{Sm} / \mathrm{La}$ vs. $\mathrm{K} / \mathrm{Nb}$ of the TRL. $\mathrm{Sm} / \mathrm{La}$ is used as a representative of the SALATHO component, whilst $\mathrm{K} / \mathrm{Nb}$ is indicative of the subduction-related signature (i.e. $\mathrm{K}_{2} \mathrm{O}$ enrichment and HFSE depletion). The observed lack of correlation suggests that the SALATHO component is not related to the process of sediment melt recycling that imparts the subduction signature to the mantle source of the TRL. Lamproite data source as in Table 1, symbols as in Figure 2.

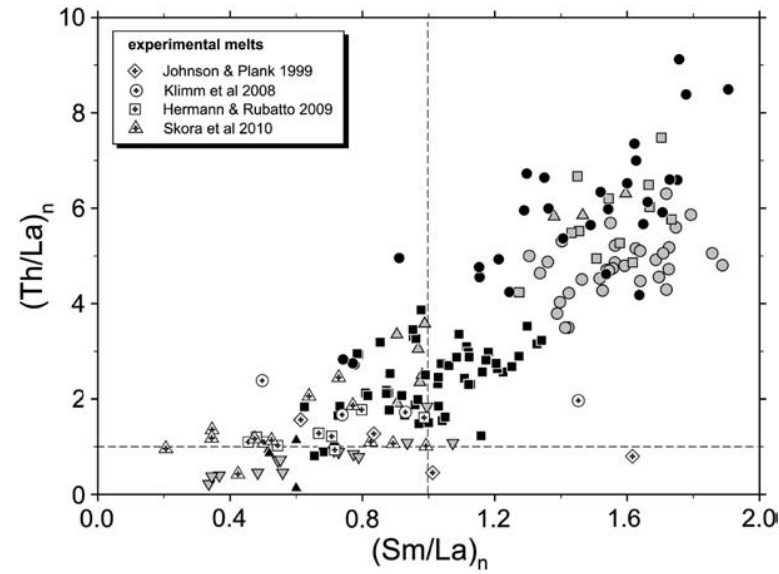

Fig. 7. $(\mathrm{Sm} / \mathrm{La})_{\mathrm{n}}$ vs. $(\mathrm{Th} / \mathrm{La})_{\mathrm{n}}$ comparison between natural (TRL) and experimental melts. In order to account for trace element doping in experimental runs, all experimental melt compositions are normalised to the composition of their starting materials. The TRL are presented normalised to GLOSS assuming that the SALATHO component is originated from a crust-like protolith (see text for details). Experimental melting of either basaltic oceanic crust (Klimm et al., 2008) or sediments (Hermann and Rubatto, 2009; Johnson and Plank, 1999; Skora and Blundy, 2010) at variable P-T conditions (both allanite/monazite saturated and undersaturated), cannot fractionate $\mathrm{Th} / \mathrm{La}$ and $\mathrm{Sm} / \mathrm{La}$ as required by the TRL. Lamproite data source as in Table 1, symbols for the TRL as in Figure 2.

history of high time-integrated $\kappa\left({ }^{232} \mathrm{Th} /{ }^{238} \mathrm{U}\right)$ and low timeintegrated $\mu\left({ }^{238} \mathrm{U} /{ }^{204} \mathrm{~Pb}\right)$. In Figure 9, we have attempted to reconstruct the $\mathrm{Pb}$ isotope composition of the SALATHO end-member assuming that it was formed from a broad crustal reservoir following $\mathrm{U} / \mathrm{Th} / \mathrm{Pb}$ fractionation occurred at different ages in the past. Thus, we first traced back to $1 \mathrm{Ga}$ the $\mathrm{Pb}$ isotope composition of a putative crust-like protolith, starting from the present day composition of GLOSS. As stated earlier, this assumption is somehow arbitrary, but it is aimed to illustrate a general scenario valid for the whole TRL. Then we applied at different ages $t(0.05,0.3,0.5,0.7,1 \mathrm{Ga}$, Fig. 9$)$, a number of $\mathrm{U} / \mathrm{Th} / \mathrm{Pb}$ fractionations, and allowed the $\mathrm{Pb}$ isotopes to age up to the time of the TRL formation. Using a trial and error approach, we have reported in Figure 9 two ideal pairs of $\kappa$ and $\mu$ (40-1.5 and 30-0.8, respectively) that can account for the $\mathrm{Pb}$ isotope array exhibited by the TRL. The short-dashed lines in Figure 9 start from the Pb isotope

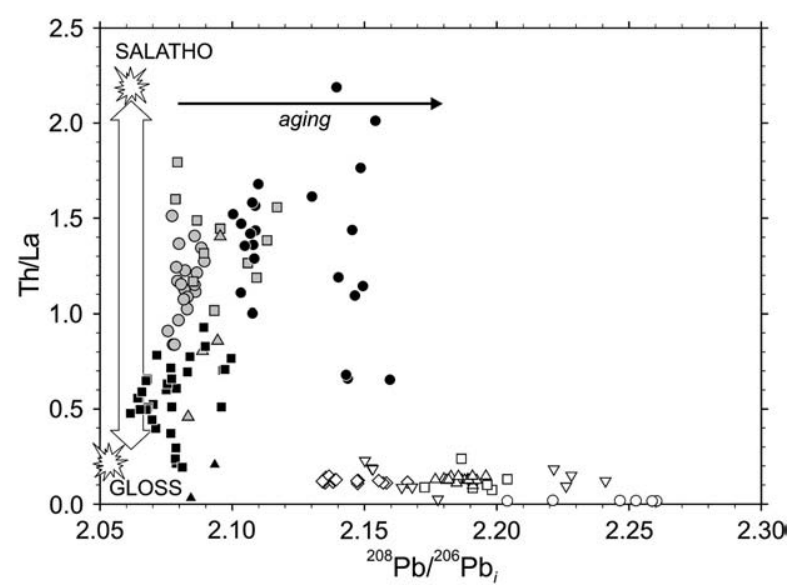

Fig. 8. ${ }^{208} \mathrm{~Pb} /{ }^{206} \mathrm{~Pb}_{i}$ vs. Th/La of the TRL and within-plate lamproites. The broad positive correlation exhibited by the TRL indicates that the metasomatic agent which causes the development of high Th/La (i.e. SALATHO) has also high ${ }^{208} \mathrm{~Pb} /{ }^{206} \mathrm{~Pb}$, indicating high time-integrated $\kappa\left({ }^{232} \mathrm{Th} /{ }^{238} \mathrm{U}\right)$. The TRL data span from a sediment melt component ( similar to GLOSS) to the SALATHO component. The horizontal spread of the data is likely to be ascribed to a variable formation age, and possibly $\kappa$, of the SALATHO component in each sector of the TRL, translating into variable ${ }^{208} \mathrm{~Pb} /{ }^{206} \mathrm{~Pb}_{i}$. Lamproite data source as in Table 1, symbols as in Figure 2. 


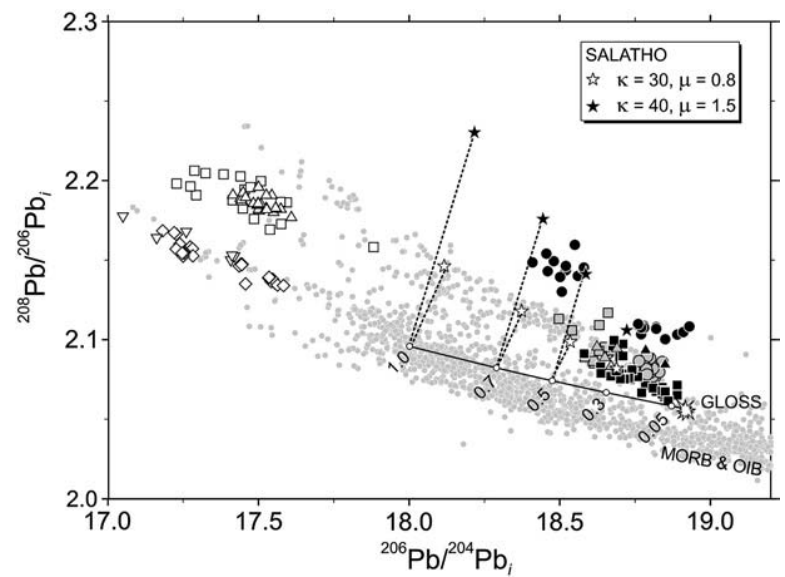

Fig. 9. ${ }^{208} \mathrm{~Pb} /{ }^{206} \mathrm{~Pb}_{i}$ vs. ${ }^{206} \mathrm{~Pb} /{ }^{204} \mathrm{~Pb}_{i}$ of the TRL and within-plate lamproites along with MORBs and OIBs (data from the compilation of Stracke et al., 2003). The TRL defines an array which deviates from that of OIBs, MORBs, and other lamproites worldwide. This requires a two component mixing between a sediment melt component (approximated by GLOSS) and another end-member characterised by high ${ }^{208} \mathrm{~Pb} /{ }^{206} \mathrm{~Pb}$ for a given ${ }^{206} \mathrm{~Pb} /{ }^{204} \mathrm{~Pb}$, indicating high time-integrated $\kappa\left({ }^{232} \mathrm{Th} /{ }^{238} \mathrm{U}\right)$ and low time-integrated $\mu$ $\left({ }^{238} \mathrm{U} /{ }^{204} \mathrm{~Pb}\right)$, respectively. This latter end-member, as suggested by Figure 8 , is identifiable with the SALATHO component originating also from a crust-like protolith. Due to the lack of more specific information on the composition of sediments in each sector of the TRL, we use GLOSS as representative of both the sediment melt endmember (i.e. low ${ }^{208} \mathrm{~Pb} /{ }^{206} \mathrm{~Pb}$ ) and as starting protolith from which the SALATHO formed at different ages in the past. Thus, in order to estimate the $\mathrm{Pb}$ isotope evolution of the SALATHO end-member, we traced back the composition of present day GLOSS (small open circles, solid line) to $0.05,0.3,0.5,0.7$, and $1.0 \mathrm{Ga}$. From each age in the past, we applied a number of $\mathrm{U} / \mathrm{Th} / \mathrm{Pb}$ fractionation and let $\mathrm{Pb}$ isotopes evolve (open and closed stars) until the formation age of the TRL. The Pb isotope evolution lines (shortdashed lines) reported in the diagram are modelled with two pairs of $\kappa$ and $\mu$ (40-1.5 and 30-0.8, respectively) suitable to fit the TRL array. For the sake of clarity we omitted the $\mathrm{Pb}$ isotope evolution lines at 50 and $300 \mathrm{Ma}$. Although the initial protolith composition is arbitrary the diagram clearly shows that the subvertical displacement of the TRL requires isolation times in the order of hundreds of millions years. This isolation time is not necessarily uniform along the Tethyan realm orogenic belts, and the higher the ${ }^{208} \mathrm{~Pb} /{ }^{206} \mathrm{~Pb}$ the older the formation age of the SALATHO end-member. Indeed, a SALATHO component formed at some $50 \mathrm{Ma}$ is barely distinguishable from the present day GLOSS, and thus cannot account for the TRL array (see text).

composition of the crustal protolith at different ages $t$, and depict the isotopic evolution of putative SALATHO components up to the formation age of the TRL, according to the two ideal pairs of $\kappa$ and $\mu$ (closed and open stars, Fig. 9). This provides information on the timescale required by potential SALATHO components to develop suitable $\mathrm{Pb}$ isotope compositions. The array exhibited by the TRL (Fig. 9), akin to Th/La vs. Sm/La (Fig. 5), suggests that their depleted mantle source peridotites have been heavily metasomatised by both normal sediment melts and the SALATHO component. The additional information provided by $\mathrm{Pb}$ isotopes is that this component must have formed at nominal ages of some 700-300 Ma.

The nominal ages determined in our model are not meant to be absolute ages because this would require an exact knowledge of the average isotope composition of the subducted sediment column in each sector of the Tethyan realm orogenic belts. The use of a sediment proxy with higher ${ }^{208} \mathrm{~Pb} /{ }^{206} \mathrm{~Pb}$ and lower ${ }^{206} \mathrm{~Pb} /{ }^{204} \mathrm{~Pb}$ than present day GLOSS would decrease the inferred nominal ages. However, the subvertical displacement exhibited by the TRL in Figure 9 provides compelling evidence for: (i) the formation of a time-integrated high $\mathrm{k}$ and low $\mu$ component, and (ii) the $\mathrm{Pb}$ isotope evolution of this component lasting for some hundreds of million years. The formation age of the SALATHO component is not necessarily contemporaneous in each sector of the TRL, although it ought to be older than the last collisional events that assembled the Tethyan realm orogenic belts. For example, it is clear from Figure 9 that a $\mathrm{U} / \mathrm{Th} / \mathrm{Pb}$ fractionation occurring at some $50 \mathrm{Ma}$ (i.e. Alpine and Himalayan orogenies) would prove very difficult to reconcile with the Pb isotope array of the TRL, for any value of $\kappa$ and $\mu$.

\section{Decoding the SALATHO component within Tethyan realm geodynamic framework}

The occurrence, albeit scattered, of the TRL all along the orogenic belts from Spain to Tibet (Fig. 1) suggests a common genetic process affecting their mantle sources. This has to be a general and widespread mechanism which characterised the development and the assemblage of the $>18,000 \mathrm{~km} \mathrm{E-W}$ striking Tethyan realm orogenic belts. The geodynamic setting is perhaps the key factor to work out the geochemical paradox of the TRL. Since the Palaeozoic, the area has been characterised by the prolonged subduction of distinct Tethyan realm oceans along with the diachronous collision of the northward drifting continental slivers from Gondwana (e.g. Hun Terrane, Variscan and Cimmerian Terranes, Gaetani et al., 2003; Scotese, 2004; Stampfli, 2000; Stampfli and Borel, 2002). The formation of tectonic mélange consisting of chaotic hybridised mixtures of peridotite, basalt, and sediment occurs in most orogenic belts, including the Tethyan realm orogenic belts (e.g. Collins and Robertson, 1997; Festa et al., 2010; Fotoohi Rad et al., 2005), and is a common by-product of the subduction cycle. Geophysical and geochemical arguments suggest the possibility that mélange domains can be an intrinsic feature of the slab-mantle interface at depth (e.g. King et al., 2006). Thus, during the final stage of closure of these ancient Tethyan realm oceanic basins and subsequent continental collision, these mélange domains along with the detached oceanic lithosphere were liable to be accreted to the innermost portions of the colliding continental plates (Prelević and Foley, 2007). Incidentally, these mélange domains have just the appropriate components (i.e. hydrothermally metamorphosed refractory peridotite and oceanic crust - basalt and sediment) required by the mantle sources of lamproites. The likely high $\mathrm{P}$ and low $\mathrm{T}$ metamorphic conditions experienced by these slices of tectonic mélanges after accretion favoured the stabilisation of metamorphic phases such as lawsonite and zoisite/epidote in the oceanic crust, in addition to hydrous $\mathrm{K}$ bearing minerals (e.g. phengite, biotite-phlogopite, amphibole).

Zoisite/epidote and lawsonite represent promising candidates to solve the geochemical conundrum of the TRL. They are a major repository of $\mathrm{Sr}, \mathrm{Pb}, \mathrm{U}, \mathrm{Th}$, and LREE and their trace element ratios (Brunsmann et al., 2000; Feineman et al., 2007; Frei et al., 2004; Hickmott et al., 1992; Spandler et al., 2003; Usui et al., 2006, and references therein), although not conclusive, seem to match those required by the SALATHO component. For example, Spandler et al. (2003) reported trace element content of lawsonite from blueschist facies pelitic samples occurring in the New Caledonia orogenic belt with $\mathrm{La}=119 \mathrm{ppm}, \mathrm{Sm}=77 \mathrm{ppm}, \mathrm{Th}=80 \mathrm{ppm}$, and $\mathrm{U}=7 \mathrm{ppm}$ (i.e. $\mathrm{Sm} / \mathrm{La}=0.64, \mathrm{Th} / \mathrm{La}=0.67, \mathrm{Th} / \mathrm{U}=11)$. Feineman et al. (2007) performed zoisite-aqueous fluid partitioning experiments at $2 \mathrm{GPa}$ and $750-900{ }^{\circ} \mathrm{C}$, and the trace element ratios of zoisite (the trace element abundances cannot be used because the starting materials were doped) formed at $900{ }^{\circ} \mathrm{C}$ were $\mathrm{Sm} / \mathrm{La}=1.17-0.89, \mathrm{Th} / \mathrm{La}=1.14$ $0.92, \mathrm{Th} / \mathrm{U}=22-80$. Admittedly, the available trace element data of lawsonite and zoisite are in places ambiguous and far from homogeneous, requiring further studies to refine their trace element signature and assess their potential role in the TRL mantle sources. For example, Th/La is not $>1.5$, but, to our knowledge, these minerals represent the closest match to the SALATHO component. The critical aspect is that these minerals must segregate from their initial protolith and subsequently melt completely to produce a metasomatic agent with high $\mathrm{Sm} / \mathrm{La}$ and $\mathrm{Th} / \mathrm{La}$. Veins and segregations formed during hydrofracturing and protolith dehydration are commonly used to deduce fluid flow in metamorphic rocks (e.g. Philippot and Selverstone, 1991) and, for example, zoisite segregations are reported in amphibolites of the Palaeozoic part of the Lower Schierferhülle, Tauern Window, Austria (Brunsmann et al., 2000). They consist of zoisite + quartz + calcite veins and segregations formed during an early to pre-Hercynian high P ( 1.5 GPa) and low 
$\mathrm{T}\left(\sim 500^{\circ} \mathrm{C}\right)$ event, and demonstrate the occurrence of this process during the assemblage of the Tethyan realm orogenic belts.

We tentatively propose a model for the formation of the TRL mantle sources that entails different time-separated stages. In a first stage, the high P and low T metamorphism experienced by the highly chaotic mélange domains accreted to the Eurasian plate determined the development of the SALATHO component (lawsonite and zoisite/ epidote veins and segregations) with characteristic trace element ratios (i.e. high $\mathrm{Sm} / \mathrm{La}$, Th/La, and $\mathrm{Th} / \mathrm{U}$ ). The $\mathrm{Pb}$ isotope composition of the TRL (Fig. 9) demands for an isolation of this component for some hundreds of million years. The isolation time may have been different in each sector of the Tethyan realm orogenic belts, in agreement with the diachronous collision of the northward drifting continental slivers from Gondwana (e.g. Hun Terrane, Variscan and Cimmerian Terranes, Gaetani et al., 2003; Scotese, 2004; Stampfli, 2000; Stampfli and Borel, 2002).

The second stage occurred during the most recent subduction events - involving, for example, the Neotethys and Alpine Tethys oceanic plate - and subsequent continental collision (Alpine, Apennine, Himalayan orogeny). The subducting sediments produced silica-rich melts that migrated through the mantle wedge and metasomatised the depleted lithospheric mantle blocks of the previously accreted chaotic mélange domains, forming a clinopyroxenephlogopite vein network (e.g. Foley, 1992b; Melzer and Foley, 2000; Sekine and Wyllie, 1982).

The third stage eventually occurred during the Tertiary orogenic belt collapses (Prelević and Foley, 2007). Lithosphere delamination and thermal relaxation triggered the onset of low-degree melting of the low-solidus fractions within the mélange domains. Lamproitic magmas were thus produced along the lines of a vein-plus-wall rock melting mechanism (Foley, 1992b) involving both the clinopyroxenephlogopite vein network and the SALATHO components. The former determined the $\mathrm{K}_{2} \mathrm{O}$ enrichment and the subduction-related signature of the TRL; the latter (lawsonite/zoisite) was totally consumed during melting producing residual garnet and kyanite (Schmidt and Poli, 2004) and imparted to the TRL their unique trace element ratios (i.e. high Sm/La and Th/La, Fig. 5).

Considering the geodynamic evolution of the Mediterranean alone (e.g. Vignaroli et al., 2008), our model opens a new scenario for the formation of the lamproite mantle source and questions the crust-like mantle (CLIMA) model of Peccerillo and Martinotti (2006), which links the formation of the lamproite mantle source of the Western Mediterranean entirely to the recent (ca. $50 \mathrm{Ma}$ ) Alpine SE-verging subduction of the European plate. In contrast, in our model the lamproite mantle source of the Mediterranean (the chaotic mélange domains) was likely formed during an earlier collisional event that piled up the northward drifted Gondwana-derived continental slivers. The successive re-opening of minor ocean basins such as the Alpine Tethys, the Adriatic-Ionic and Vardar oceans (e.g. Stampfli, 2000; Stampfli and Borel, 2002; Vignaroli et al., 2008) broke-up the accreted continental plates and the tectonic mélange domains. Later, in response to the Alpine and Apennine orogenic belt collapses, these tectonic mélange domains were melted during the recent magmatic activity which characterised the Mediterranean area from Spain to Anatolia (Fig. 1) and produced small-volume lamproitic magmas.

Supplementary materials related to this article can be found online at doi:10.1016/j.epsl.2010.11.023.

\section{Acknowledgements}

We would like to thank Steve Foley, Cliff Shaw, and an anonymous reviewer for their constructive and thorough criticism. Focusing and stirring the discussion on the geodynamic evolution of the Mediterranean-Himalayan system with Massimo Mattei, Federico Sani, and Claudio Faccenna is greatly appreciated. The criticism of Eleonora Braschi on an early draft of the manuscript is acknowledged. We thank Dejan Prelević for sharing his database on the Vardar lamproites, and Susanne Skora for helpful discussion on her results on sediment melting in subduction zones. This work was supported by the Italian Ministry for Research and Education (MIUR) grant (PRIN 2008, \# 2008HMHYFP_002) issued to S.C., and by the "Rientro dei Cervelli" project granted to R.A.

\section{References}

Avanzinelli, R., Lustrino, M., Mattei, M., Melluso, L., Conticelli, S., 2009. Potassic and ultrapotassic magmatism in the circum-Tyrrhenian region: Significance of carbonated pelitic vs. pelitic sediment recycling at destructive plate margins. Lithos $113,213-227$

Altherr, R., Meyer, H.P., Holl, A., Volker, F., Alibert, C., McCulloch, M.T., Majer, V., 2004 Geochemical and $\mathrm{Sr}-\mathrm{Nd}-\mathrm{Pb}$ isotopic characteristics of Late Cenozoic leucite lamproites from the East European Alpine belt (Macedonia and Yugoslavia). Contrib. Mineralog. Petrol. 147, 58-73.

Altherr, R., Topuz, G., Siebel, W., Şen, C., Meyer, H.-P., Satır, M., Lahaye, Y., 2008. Geochemical and $\mathrm{Sr}-\mathrm{Nd}-\mathrm{Pb}$ isotopic characteristics of Paleocene plagioleucitites from the Eastern Pontides (NE Turkey). Lithos 105, 149-161.

Argand, E., 1924. La Tectonique de L'Asie. Compte Rendu du XIII Congrès Géologique International, Brussel, pp. 171-372.

Benito, G.R., Lopez Ruiz, J., Cebria, J.M., Hertogen, J., Doblas, M., Oyarzun, R., Demaiffe, D., 1999. Sr and O isotope constraints on source and crustal contamination in the High-K calc-alkaline and shoshonitic Neogene volcanic rocks of SE Spain. Lithos $46,773-802$

Bergman, S.C., 1987. Lamproites and other potassium-rich igneous rock, a review of their occurrences, mineralogy and geochemistry. In: Fitton, J.G., Upton, B.G.J. (Eds.), Alkaline Igneous Rocks: Geological Society of London, Special Publications, 30, pp. 103-189.

Brunsmann, A., Franz, G., Erzinger, J., Landwehr, D., 2000. Zoisite- and clinozoisitesegregations in metabasites (Tauern Window, Austria) as evidence for highpressure fluid-rock interaction. J. Metamorph. Geol. 18, 1-22.

Chung, S.L., Chu, M.F., Zhang, Y., Xie, Y., Lo, C.H., Lee, T.Y., Lan, C.Y., Li, X., Zhang, Q., Wang, Y., 2005. Tibetan tectonic evolution inferred from spatial and temporal variations in post-collisional magmatism. Earth Sci. Rev. 68, 173-196.

Coban, H., Flower, M.F.J., 2007. Late Pliocene lamproites from Bucak, Isparta (southwestern Turkey): implications for mantle "wedge" evolution during Africa-Anatolia plate convergence. J. Asian Earth Sci. 29, 160-176.

Collins, A.S., Robertson, A.H.F., 1997. Lycian melange, southwestern Turkey: an emplaced Late Cretaceous accretionary complex. Geology 25, 255-258.

Conticelli, S., Peccerillo, A., 1992. Petrology and geochemistry of potassic and ultrapotassic alkalic volcanism in Central Italy: petrogenesis and interferences on the mantle source. Lithos 28, 21-240.

Conticelli, S., Carlson, R.W., Widow, E., Serri, G., 2007. Chemical and isotopic composition $(\mathrm{Os}, \mathrm{Pb}, \mathrm{Nd}$, and $\mathrm{Sr}$ ) of Neogene to Quaternary calc-alkaline, shoshonitic, and ultrapotassic mafic rocks from the Italian peninsula: Inferences on the nature of their mantle sources. In: Beccaluva, L., Bianchini, G., Wilson, M. (Eds.), Cenozoic Volcanism in the Mediterranean Area: Geological Society of America, Special Papers, 418, pp. 171-202.

Conticelli, S., Guarnieri, L., Farinelli, A., Mattei, M., Avanzinelli, R., Bianchini, G., Boari, E., Tommasini, S., Tiepolo, M., Prelević, D., Venturelli, G., 2009. Trace elements and $\mathrm{Sr}-\mathrm{Nd}-\mathrm{Pb}$ isotopes of K-rich, shoshonitic, and calc-alkaline magmatism of the Western Mediterranean Region: genesis of ultrapotassic to calcalkaline magmatic associations in a post-collisional geodynamic setting. Lithos 107, 68-92.

Cvetković, V., Prelević, D., Downes, H., Jovanovic, M., Vaselli, O., Pecskay, Z., 2004. Origin and geodynamic significance of Tertiary postcollisional basaltic magmatism in Serbia (Central Balkan Peninsula). Lithos 73, 161-186.

Davies, G.R., Stolz, A.J., Mahotkin, I.L., Nowell, G.M., Pearson, D.G., 2006. Trace element and $\mathrm{Sr}-\mathrm{Pb}-\mathrm{Nd}-\mathrm{Hf}$ isotope evidence for ancient, fluid-dominated enrichment of the source of Aldan Shield lamproites. J. Petrol. 47, 1119-1146.

Dilek, Y., Altunkaynak, S., 2007. Cenozoic crustal evolution and mantle dynamics of post-collisional magmatism in Western Anatolia. Int. Geol. Rev. 49, 431-453.

Duggen, S., Hörnle, K., van den Bogaard, P., Harris, C., 2004. Magmatic evolution of the Alboran region: the role of subduction in forming the Western Mediterranean and causing the Messinian salinity crisis. Earth Planet. Sci. Lett. 218, 91-108.

Edgar, A.D., 1987. The genesis of alkaline magmas with emphasis on their source regions: inferences from experimental studies. In: Fitton, J.G., Upton, B.G.J. (Eds.), Alkaline Igneous Rocks: Geological Society of London, Special Publication, 30, pp. 29-52.

Edgar, A.D., Vukadinovic, D., 1992. Implications of experimental petrology to the evolution of ultrapotassic rocks. Lithos 28, 205-220.

Ersoy, Y., Helvaci, C., 2007. Stratigraphy and geochemical features of the Early Miocene bimodal (ultrapotassic and calc-alkaline) volcanic activity within the NE-trending Selendi Basin, Western Anatolia, Turkey. Turk. J. Earth Sci. 16, 117-139.

Feineman, M.D., Ryerson, F.J., DePaolo, D.J., Plank, T., 2007. Zoisite-aqueous fluid trace element partitioning with implications for subduction zone fluid composition. Chem. Geol. 239, 250-265.

Festa, A., Pini, G.A., Dilek, Y., Codegone, G., Vezzani, L., Ghisetti, F., Lucente, C.C., Ogata, K., 2010. Peri-Adriatic melanges and their evolution in the Tethyan realm. Int. Geol. Rev. 52, 369-403.

Foley, S.F., 1992a. Petrological characterisation of the source components of potassic magmas: geochemical and experimental constraints. Lithos 28, 187-204. 
Foley, S.F., 1992b. Vein-plus-wall-rock melting mechanisms in the lithosphere and the origin of potassic alkaline magmas. Lithos $28,435-453$.

Fotoohi Rad, G.R., Droop, G.T.R., Amini, S., Moazzen, M., 2005. Eclogites and blueschists of the Sistan Suture Zone, eastern Iran: a comparison of P-T histories from a subduction mélange. Lithos 84, 1-24.

Francalanci, L., Innocenti, F., Manetti, P., Savasçin, M.Y., 2000. Neogene alkaline volcanism of the Afyon-Isparta area, Turkey: petrogenesis and geodynamic implications. Mineral. Petrol. 70, 285-312.

Fraser, K.J., Hawkesworth, C.J., Erlank, A.J., Mitchell, R.H., Scott-Smith, B.H., 1985. Sr, Nd and $\mathrm{Pb}$ isotope and minor element geochemistry of lamproites and kimberlites. Earth Planet. Sci. Lett. 76, 57-70.

Frei, D., Liebscher, A., Franz, G., Dulski, P., 2004. Trace element geochemistry of epidote minerals. Rev. Mineral. Geochem. 56, 553-605.

Gaetani, M., Dercourt, J., Vrielynck, B., 2003. The peri-tethys programme: achievements and results. Episodes 26, 79-93.

Gao, Y., Hou, Z., Kamber, B.S., Wei, R., Meng, X., Zhao, R., 2007. Lamproitic rocks from a continental collision zone: evidence for recycling of subducted Tethyan oceanic sediments in the mantle beneath Southern Tibet. J. Petrol. 48, 729-752.

Harangi, S., Downes, H., Seghedi, I., 2006. Tertiary-Quaternary subduction processes and related magmatism in the Alpine-Mediterranean region. Geol. Soc. Lond. Mem. 32, 167-190.

Hermann, J., Rubatto, D., 2009. Accessory phase control on the trace element signature of sediment melts in subduction zones. Chem. Geol. 265, 512-526.

Hickmott, D.D., Sorensen, S.S., Rogers, P.S.Z., 1992. Metasomatism in a subduction complex: constraints from microanalysis of trace elements in minerals from garnet amphibolite from the Catalina Schist. Geology 20, 347-350.

Innocenti, F., Agostini, S., di Vincenzo, G., Doglioni, C., Manetti, P., Savascin, M.Y. Tonarini, S., 2005. Neogene and Quaternary volcanism in Western Anatolia: magma sources and geodynamic evolution. Mar. Geol. 221, 397-421.

Johnson, M.C., Plank, T., 1999. Deydratation and melting experiments constraints the fate of subducted sediments. Geochem. Geophys. Geosyst. 1 paper no. 1999GC000014

Kessel, R., Schmidt, M.W., Ulmer, P., Pettke, T., 2005. Trace element signature of subduction-zone fluids, melts and supercritical liquids at 120-180 km depth. Nature 437, 724-727.

King, R.L., Bebout, G.E., Moriguti, T., Nakamura, E., 2006. Elemental mixing systematics and $\mathrm{Sr}-\mathrm{Nd}$ isotope geochemistry of melange formation: obstacles to identification of fluid sources to arc volcanics. Earth Planet. Sci. Lett. 246, 288-304.

Klimm, K., Blundy, J.D., Green, T.H., 2008. Trace element partitioning and accessory phase saturation during $\mathrm{H}_{2} \mathrm{O}$-saturated melting of basalt with implications for subduction zone chemical fluxes. J. Petrol. 49, 523-553.

Lustrino, M., Wilson, M., 2007. The circum-Mediterranean anorogenic Cenozoic igneous province. Earth Sci. Rev. 81, 1-65.

McDonough, W.F., 1990. Constraints on the composition of the continental lithospheric mantle. Earth Planet. Sci. Lett. 101, 1-18.

Melzer, S., Foley, S.F., 2000. Phase relations and fractionation sequences in potassic magma series modelled in the system $\mathrm{CaMgSi}_{2} \mathrm{O}_{6}-\mathrm{KAlSiO}_{4}-\mathrm{Mg}_{2} \mathrm{SiO}_{4}-\mathrm{F}_{2} \mathrm{O}_{-1}$ at $1 \mathrm{bar}$ to 18 kbar. Contrib. Mineralog. Petrol. 138, 186-197.

Miller, C., Schuster, R., Klotzli, U., Frank, W., Purtscheller, F. 1999. Post-collisional potassic and ultrapotassic magmatism in SW Tibet: geochemical and $\mathrm{Sr}-\mathrm{Nd}-\mathrm{Pb}-\mathrm{O}$ isotopic constraints for mantle source characteristics and petrogenesis. J. Petrol. 40, 1399-1424.

Mirnejad, H., Bell, K., 2006. Origin and source evolution of the Leucite Hills lamproites: evidence from Sr-Nd-Pb-O isotopic compositions. J. Petrol. 47, 2463-2489.

Mitchell, R.H., Platt, R.G., Downey, M., 1987. Petrology of lamproites from Smoky Butte, Montana. J. Petrol. 28, 645-677.

Murphy, D.T., Collerson, K.D., Kamber, B.S., 2002. Lamproites from Gaussberg, Antarctica: possible transition zone melts of Archaean subducted sediments. J. Petrol. 43, 981-1001.

Nelson, D.R., McCulloch, M.T., Sun, S.-S., 1986. The origins of ultrapotassic rocks as inferred from Sr, Nd and Pb isotopes. Geochim. Cosmochim. Acta 50, 231-245.

Owen, J.P., 2008. Geochemistry of lamprophyres from the Western Alps, Italy: implications for the origin of an enriched isotopic component in the Italian mantle. Contrib. Mineralog. Petrol. 155, 341-362.

Peccerillo, A., Martinotti, G., 2006. The Western Mediterranean lamproitic magmatism: origin and geodynamic significance. Terra Nova 18, 109-117.

Philippot, P., Selverstone, J., 1991. Trace-element-rich brines in eclogitic veins: implications for fluid composition and transport during subduction. Contrib. Mineralog. Petrol. 106, 417-430.

Plank, T., 2005. Constraints from thorium/lanthanum on sediment recycling at subduction zones and the evolution of the continents. J. Petrol. 46, 921-944.

Plank, T., Langmuir, C.H., 1998. The chemical composition of subducting sediments and its consequence for the crust and mantle. Chem. Geol. 145, 325-394.
Prelević, D. Foley, S.F., 2007. Accretion of arc-oceanic lithospheric mantle in the Mediterranean: evidence from extremely high-Mg olivines and $\mathrm{Cr}$-rich spinel inclusions from lamproites. Earth Planet. Sci. Lett. 256, 120-135.

Prelević, D., Foley, S.F., Cvetković, V., Romer, R.L., 2004. Origin of minette by mixing of lamproite and dacite magmas in Veliki Majdan Serbia. J. Petrol. 45, 759-792.

Prelević, D., Foley, S.F., Romer, R.L., Cvetković, V., Downes, H., 2005. Tertiary ultrapotassic volcanism in Serbia: constraints on petrogenesis and mantle source characteristics. J. Petrol. 46, 1443-1487.

Prelević, D., Foley, S.F., Romer, R.L., Conticelli, S., 2008. Mediterranean Tertiary lamproites: multicomponent melts in post-collisional geodynamics. Geochim. Cosmochim. Acta 72, 2125-2156.

Prowatke, S., Klemme, S., 2006. Trace element partitioning between apatite and silicate melts. Geochim. Cosmochim. Acta 70, 4513-4527.

Rudnick, R.L., Gao, S., 2003. Composition of the continental crust, in: The Crust, Rudnick, R.L. (Ed.) 3, 1-64 of Treatise on Geochemistry, H. D. Holland, H.D., and Turekian, K.K. (Eds.), Elsevier-Pergamon, Oxford.

Schmidt, M.W., Poli, S., 2004. Magmatic epidote. Rev. Mineral. Geochem. 56, 399-430.

Scotese, C.R., 2004. A continental drift flipbook. J. Geol. 112, 729-741.

Sekine, T., Wyllie, P.J., 1982. Phase relationships in the system KAlSiO4- Mg2SiO4 $\mathrm{SiO} 2-\mathrm{H} 2 \mathrm{O}$ as a model for hybridization between hydrous siliceous melts and peridotite. Contrib. Mineralog. Petrol. 79, 368-374.

Skora, S., Blundy, J., 2010. High-pressure hydrous phase relations of radiolarian clay and implications for the involvement of subducted sediment in arc magmatism. J. Petrol. doi:10.1093/petrology/egq054, online version.

Spandler, C., Hermann, J., Arculus, R., Mavrogenes, J., 2003. Redistribution of trace elements during prograde metamorphism from lawsonite blueschist to eclogite facies; implications for deep subduction-zone processes. Contrib. Mineralog. Petrol. 146, 205-222.

Stampfli, G.M., 2000. Tethyan Oceans. In: Bozkurt, E., Winchester, J.A., Piper, P.A. (Eds.), Geological Society of London, Special Publication, 173, pp. 1-23.

Stampfli, G.M., Borel, G.D., 2002. A plate tectonic model for the Paleozoic and Mesozoic constrained by dynamic plate boundaries and restored synthetic oceanic isochrons. Earth Planet. Sci. Lett. 196, 17-33.

Stracke, A., Bizimis, M., Salters, J.M., 2003. Recycling oceanic crust: quantitative constraints. Geochem. Geophys. Geosyst. 4 (3), 8003. doi:10.1029/2001GC000223.

Suess, E., 1893. Are great ocean depths permanent? Nat. Sci. 2, 180-187.

Sun, S.-S., McDonough, W.F., 1989. Chemical and isotopic systematics of Oceanic Basalts: implications for mantle composition and processes. In: Saunders, A.D. Norry, M.J. (Eds.), Magmatism in the Ocean Basins: Geological Society of London, Special Publication, 42, pp. 313-345.

Topuz, G., Altherr, R., Schwarz, W.H., Siebel, W., Satır, M., Dokuz, A., 2005. Postcollisional plutonism with adakite-like signatures: the Eocene Saraycık granodiorite (Eastern Pontides, Turkey). Contrib. Mineralog. Petrol. 150, 441-455.

Turner, S.P., Arnaud, N., Liu, J., Rogers, N., Hawkesworth, C.J., Harris, N., Kelley, S., van Calsteren, P., Deng, W., 1996. Post-collision, shoshonitic volcanism on the Tibetan Plateau: implications for convective thinning of the lithosphere and the source of ocean island basalts. J. Petrol. 37, 45-61.

Usui, T., Nakamura, E., Helmstaedt, H., 2006. Petrology and geochemistry of eclogite xenoliths from the Colorado plateau: implications for the evolution of subducted oceanic crust. J. Petrol. 47, 929-964.

Vignaroli, G., Faccenna, C., Jolivet, L., Piromallo, C., Rossetti, F., 2008. Subduction polarity reversal at the junction between the Western Alps and the Northern Apennines, Italy. Tectonophysics 450, 34-50.

Vollmer, R., Ogden, P., Schilling, J.G., Kingsley, R.H., Waggoner, D.G., 1984. Nd and Sr isotopes in ultrapotassic volcanic rocks from the Leucite Hills, Wyoming. Contrib. Mineralog. Petrol. 87, 359-368.

Wendlandt, R.F., Eggler, D.H., 1980a. The origin of potassic magmas: 1. Melting relations in the systems $\mathrm{KAlSiO}_{4}-\mathrm{Mg}_{2} \mathrm{SiO}_{4}-\mathrm{SiO}_{2}$ and $\mathrm{KAISiO}_{4}-\mathrm{MgO}-\mathrm{SiO}_{2}-\mathrm{CO}_{2}$ to $30 \mathrm{~kb}$. Am. J. Sci. 280, 385-420.

Wendlandt, R.F., Eggler, D.H., 1980b. The origin of potassic magmas: 2. Stability of phlogopite in natural spinel lherzolite and in the system $\mathrm{KAlSiO}_{4}-\mathrm{Mg}_{2} \mathrm{SiO}_{4}-\mathrm{SiO}_{2}$ $\mathrm{H}_{2} \mathrm{O}-\mathrm{CO}_{2}$ at high pressure and high temperature. Am. J. Sci. 280, 421-458.

Williams, H.M., Turner, S.P., Pearce, J.A., Kelley, S.P., Harris, N.B.W., 2004. Nature of the source regions for post-collisional, potassic magmatism in southern and northern Tibet from geochemical variations and inverse trace element modelling. J. Petrol. 45, 555-607.

Wilson, M., Bianchini, G., 1999. Tertiary-Quaternary extension-related alkaline magmatism within the Mediterranean and surrounding regions. In: Durand, B. Jolivet, L., Horvath, F., Séranne, M. (Eds.), Geological Society of London, Special Publication, 156, pp. 141-168. 